\author{
Luigi Mariano Guzzo \\ (assegnista di ricerca in Diritto ecclesiastico e canonico nell'Università degli Studi \\ "Magna Graecia" di Catanzaro, Dipartimento di Giurisprudenza, Economia e \\ Sociologia)
}

\title{
Prime annotazioni sull'Intes $a$ tra Repubblica italiana e Santa Sede per l'assistenza spirituale ai militari cattolici: una riforma gattopardesca *
}

Some comments on the agreement between the Italian Republic and the Holy See for spiritual assistance to the Catholic members of the Armed Forces*

ABSTRACT: Through the application of the Italian Law no. 70/2021, we see the long-awaited revision of the institute of religious assistance to Catholics within the Armed Forces, with the authorization of the ratification of the Exchange of Letters between the President of the Council of Ministers and the Secretary of the Vatican City State (on February 13, 2018) and the related rules for the adaptation of the internal legal system. The new aspects, however, are very limited in scope.

SOMMARIO: 1. Premessa - 2. La forma: la qualificazione dell'Intesa come accordo di natura internazionalistica - 3. La ratifica e l'esecuzione dell'Intesa nell'ordinamento dello Stato, con le relative norme di adeguamento: l'esito paradossale di una disciplina "duplicata" - 4. Le ondivaghe "vicissitudini" dell'art. 17 C.O.M.: un "termostato" delle tensioni sociali e giuridiche riguardanti la materia e l'esigenza di una riforma - 5. Politica concordataria della Santa Sede e attribuzioni alle Conferenze episcopali nazionali. Alcune considerazioni sulla polarizzazione del dibattito pubblico in Italia - 6. I contenuti dell'Intesa; 7. Le norme di adeguamento dell'ordinamento interno: novità e rilievi critici - 7.1. La direzione e il coordinamento del Servizio di assistenza spirituale: una titolarità giuridica diretta in capo all'Ordinario militare per l'Italia - 7.2. L'introduzione della figura dei Cappellani militari coordinatori, in luogo degli Ispettori: alcuni problemi di sicurezza per le istituzioni militari dello Stato- 7.3. Il concetto canonico di "sede vacante" entra nell'ordinamento militare - 7.4. La procedura di individuazione e di determinazione delle sedi per i cappellani militari - 7.5. L'art. 1533-bis C.O.M.: il divieto di corresponsione di emolumenti accessori e la materia "spirituale e pastorale" - 7.6. La nomina dell'Ordinario militare e del Vicario generale - 7.7. Il regime di assimilazione ai gradi gerarchici per i cappellani militari: nella sostanza non cambia nulla - 7.8. Altre novelle legislative: 1'organico dei cappellani; la procedura di nomina; la dismissione dallo stato clericale - 8 . Prime tensioni tra Ordinariato militare e amministrazione della Difesa sull'applicazione della legge - 9. Perché nell'Intesa non c'è alcun riferimento alle donne consacrate che operano negli stabilimenti militari? - 10. Conclusioni: il Capo I della legge n. 70 del 2021 come "caso-studio" del decadimento normativo e della crisi del Parlamento. 


\section{1 - Premessa}

La revisione dell'istituto ${ }^{1}$ del servizio di assistenza religiosa alle Forze armate per i cattolici ${ }^{2}$ è operata tramite la legge n. 70 del 22 aprile 2021 (pubblicata sulla Gazzetta Ufficiale n. 121 del 2021) che comprende, al Capo I, l'autorizzazione alla ratifica dello Scambio di Lettere - avvenuto in data 13 febbraio 2018 - tra l'allora Presidente del Consiglio dei Ministri Paolo Gentiloni e il Segretario di Stato della Città del Vaticano, card. Pietro Parolin, contenente la procedura attuativa dell'art. 11, secondo comma, dell'Accordo del 1984, insieme alle norme di adeguamento dell'ordinamento interno, nonché, al Capo II, altre disposizioni relative all'informazione del pubblico ministero sull'esercizio dell'azione penale nei confronti degli ecclesiastici ${ }^{3}$ e al diritto dei cardinali di rendere testimonianza in luogo diverso da quello previsto dalle leggi ${ }^{4}$.

In questo contributo annoteremo il Capo I della richiamata legge, relativo, quindi, all'assistenza spirituale alle Forze armate, che opera un riassetto normativo in materia del d.lgs.vo n. 66 del 25 marzo 2010 (Codice dell'ordinamento militare ${ }^{5}$ ), modificandone alcuni articoli.

Com'è noto, nonostante l'art. 11 dell'Accordo del 1984 abbia previsto che l'assistenza religiosa "alle forze armate, alla polizia o ad altri

* Contributo sottoposto a valutazione - Article peer evaluated.

${ }^{1} \mathrm{Mi}$ sono occupato dell'istituto dell'assistenza religiosa alle Forze armate, soprattutto in chiave di diritto europeo e di diritto comparato, nel volume L.M. GUZZO, L'assistenza religiosa alle Forze armate nello spazio giuridico europeo. Principi generali e analisi comparata in Italia, Regno Unito, Francia e Spagna, Wolters Kluwer-Cedam, Milano, 2019, al quale rimando per la bibliografia ivi contenuta alle pp. 193-221.

2 Sul processo di revisione in atto che sta interessando l'assistenza religiosa alle Forze armate per i cattolici si rinvia, per tutti, a P. CAVANA, Cappellani militari e prospettive di riforma, in Stato, Chiese e pluralismo confessionale, Rivista telematica (https://www.statoechiese.it), n. 9 del 2016; L.M. GUZZO, Verso la riforma dell'assistenza religiosa per $i$ cattolici nelle Forze armate, in Stato, Chiese e pluralismo confessionale, cit., n. 36 del 2016; A. DE OTO, Altare e mostrine. Contributo allo studio dell'evoluzione normativa del servizio di assistenza spirituale ai militari in Italia, Wolters Kluwer-Cedam, Milano, 2018; A. DE OTO, Il servizio di assistenza spirituale ai militari. Tra diritto confessionale e diritto dello Stato, Bononia University Press, Bologna, 2020.

3 Scambio di Lettere tra il Presidente del Consiglio dei Ministri e il Segretario di Stato della Santa Sede del 26 luglio 2006.

4 Scambio di Lettere tra il Presidente del Consiglio dei Ministri e il Segretario di Stato della Santa Sede del 15 febbraio 2008.

${ }^{5}$ D'ora in avanti, per brevità, "C.O.M." o "Codice". 
servizi assimilati" deve essere assicurata secondo "lo stato giuridico, l'organico e le modalità stabiliti d'intesa" fra le autorità italiane competenti e l'autorità ecclesiastica, soltanto per la Polizia di Stato ("smilitarizzata" con legge n. 121 del 1981), prima della legge in commento, aveva trovato attuazione la disposizione concordataria con il d.p.r. n. 421 del 1999 che ha dato esecuzione all'Intesa firmata il 9 settembre 1999 tra il Ministero dell'Interno e la Conferenza Episcopale Italiana ${ }^{6}$.

Per le Forze armate e i Corpi di polizia a ordinamento militare, anche dopo l'entrata in vigore del Codice dell'ordinamento militare (d.lgs.vo n. 66 del 2010), il servizio di assistenza religiosa continuava a essere disciplinato unilateralmente dal diritto statale. In realtà, sebbene unilaterale, si trattava comunque di una normativa chiaramente di favore per la Chiesa cattolica, rispetto alle altre confessioni religiose, che non pochi problemi di natura interpretativa ha comportato alla luce del supremo principio di laicità dello Stato ${ }^{7}$, pure soltanto a considerare come i cappellani cattolici fossero gerarchicamente inquadrati nell'organizzazione militare - tramite l'instaurazione di un rapporto di pubblico impiego -, quando, al contrario, la prestazione religiosa degli altri ministri di culto era (ed è) soggetta, di volta in volta, sulla base delle richieste, ad autorizzazione della competente autorità militare, con i relativi oneri finanziari del servizio di assistenza a carico dei gruppi confessionali ${ }^{8}$.

A fronte dell'art. 1471, primo comma, C.O.M. circa il diritto dei militari di "esercitare il culto di qualsiasi religione e ricevere l'assistenza dei militari", nel dettaglio il Codice regolamentava al Titolo III del Libro V (artt. 1533-1625 C.O.M.) solo il Servizio di assistenza spirituale per il personale militare di religione cattolica, la cui disciplina rimaneva ferma "fino all'entrata in vigore dell'intesa prevista all'art. 11, secondo comma, dell'Accordo", a norma dell'art. 17 C.O.M.

${ }^{6}$ Sul tema cfr. N. FIORITA, Brevi considerazioni sulla Intesa riguardante l'assistenza spirituale al Personale della Polizia di Stato, in Quaderni di diritto e politica ecclesiastica, $\mathrm{n}$. 2/2000, pp. 437-444; R. PASCALI, La parabola dell'assistenza spirituale alla Polizia di Stato (nella critica delle fonti), Giappichelli, Torino, 2012.

7 Per tutti, A. VALSECCHI, L'assistenza spirituale nelle comunità separate, in G. CASUSCELli (a cura di), Nozioni di Diritto ecclesiastico, 5a ed., Giappichelli, Torino, 2015, p. 212.

${ }^{8}$ Ad esempio, l'Intesa stipulata con le chiese rappresentate dalla Tavola valdese (legge n. 449 del 1984) all'art. 5, quinto comma, espressamente dispone che gli oneri finanziari per lo svolgimento dell'assistenza spirituale nelle Forze armate sono a carico degli "organi ecclesiastici competenti". 
Non si trattava, però, semplicemente di una questione di adempimento di obblighi concordatari. L'esigenza di intervenire sul servizio di assistenza religiosa, si inseriva, infatti, in un ampio processo di riforma delle Forze armate, caratterizzato, in particolare, dall'entrata in servizio di personale femminile (legge n. 380 del 1999) e dall'abolizione della coscrizione obbligatoria (legge n. 226 del 2004)9. Una revisione dell'istituto si rendeva così sempre più urgente e non più rinviabile.

\section{2 - La forma: 1'Intesa come accordo di natura internazionalistica}

Per la revisione dell'istituto dell'assistenza religiosa ai cattolici nelle Forze armate si è intrapresa la strada di un trattato internazionale - che le Parti qualificano, nel rispetto del principio della "libertà della forma", come Intesa ${ }^{10}$-, tra Governo italiano e Santa Sede, tramite lo Scambio di note diplomatiche.

D'altronde, come è stato sostenuto in dottrina:

"Nel quadro della varietà terminologica esistente nell'esperienza concordataria, si possono distinguere - anche ai sensi del can. 365 del codex iuris canonici del 1983 - i "concordati" in senso stretto (accordi stipulati in forma solenne con cui viene regolamentata la generalità delle questioni di comune interesse), dalle "altre convenzioni similari" diversamente denominate (accordi, patti, convenzioni, intese, modus vivendi, protocolli, scambi di note, scambi di lettere, etc.) costituenti strumenti negoziali più limitati nella forma (in quanto stipulati attraverso procedimenti diplomatici semplificati) o nella sostanza (in ragione del loro circoscritto contenuto materiale). Tuttavia, la possibile differente denominazione non incide sulla natura giuridica delle diverse convenzioni stipulate fra la Chiesa cattolica e gli Stati a livello istituzionale apicale, ossia quando esse risultano negoziate e sottoscritte da organi di vertice delle due Parti come, appunto, la Santa Sede e i diversi Governi statuali. Nella specie, dal punto di vista sistematico, anche per le particolari qualità soggettive delle Parti stipulanti, esse hanno, tutte, natura giuridica

${ }^{9}$ Cfr. P. CONSORTI, Cappellani militari e riforma delle forze armate, in Aggiornamenti Sociali, n. 5/1999, p. 365 ss.

$10 \mathrm{Si}$ ricordi, peraltro, che a norma della Convenzione di Vienna sul diritto dei trattati, del 1969, «il termine "trattato" indica un accordo internazionale concluso per iscritto tra Stati e regolato dal diritto internazionale, che sia costituito da un solo strumento o da due o più strumenti connessi, qualunque ne sia la particolare denominazione» (art. 2, primo comma, lett. a). 
internazionale e vincolano reciprocamente $\mathrm{i}$ soggetti contraenti all'osservanza degli impegni bilateralmente assunti (pacta sunt servanda)"11.

Un accordo internazionale, insomma, che comporta "modificazioni di legge", e la cui ratifica da parte del Presidente della Repubblica deve essere autorizzata con legge, ai sensi dell'art. 80 Cost. ${ }^{12}$.

Nello specifico, il testo è stato redatto da una Commissione paritetica istituita, ai sensi dell'art 14 dell'Accordo del 1984, nel 2015 dal Governo italiano e dalla Santa Sede ${ }^{13}$, che l'ha approvato il 18 dicembre 2017, per portarlo così alla firma delle due Parti14 il 13 febbraio 2018, a Roma e nella Città del Vaticano. L'accordo è poi confluito nel Capo I (dall'art. 1 all'art. 3) di un disegno di legge, approvato dal Consiglio dei Ministri, presentato al Senato in data 10 marzo 2020, e assegnato alla terza Commissione permanente "Affari esteri, immigrazione" con una richiesta di parere indirizzata alla quarta Commissione "Difesa", per essere infine approvato a Palazzo Madama il successivo 9 settembre. Alla Camera dei deputati si è provveduto a unire le due Commissioni in sede referente (con la richiesta di pareri alle Commissioni "Affari costituzionali", "Giustizia" e "Bilancio")15, con l'approvazione definitiva il 14 aprile 2021.

Com'è noto, dal periodo prerepubblicano successivo alla stipula dei Patti Lateranensi del 1929 fino ai nostri giorni, il procedimento dello Scambio di note fra Governo e Santa Sede, produttore di obblighi internazionali, è stato ampiamente utilizzato, anche per la regolamentazione dei rapporti giuridici su questioni inerenti il rapporto tra lo Stato e la Chiesa in Italia ${ }^{16 .}$

11 P. LILLO, Note preliminari sui protocolli concordatari, in Stato, Chiese e pluralismo confessionale, cit., n. 41 del 2013, p. 8.

12 Per alcuni primi commenti dottrinali si rimanda a G. DALLA TORRE, Nuova intesa sull'assistenza religiosa ai militari in Italia, in Archivio Giuridico, n. 2/2018, p. 413; A. DE OTO, Il servizio di assistenza spirituale, cit., p. 116 ss.; L.M. GUZZO, Riforma dei cappellani militari? , in Il Regno - Attualità, n. 20/2021, p. 630.

13 La Commissione paritetica è stata istituita dal Governo italiano e dalla Santa Sede con Scambio di Lettere in data 30 giugno 2015 e 23 ottobre 2015.

14 Il Consiglio dei Ministri del Governo pro tempore ha approvato l'intesa nella seduta dell'8 febbraio 2018.

15 Per quanto riguarda l'individuazione delle Commissioni, bisogna ricordare che il disegno di legge in esame contiene, come già detto in Premessa (vedi supra par. 1), oltre alla disciplina sull'assistenza religiosa alle Forze armate, anche altre norme di adeguamento dell'ordinamento interno a obbligazioni internazionali contratte con la Santa Sede, che attengono a materie diverse rispetto a quelle dell'assistenza religiosa.

${ }^{16}$ Cfr., per tutti, Scambio di Note circa il trasferimento dei privilegi dell'estraterritorialità 
dall'antico Palazzo dei Convertendi a quello ricostruito in Via della Conciliazione, 25 gennaio 1937 - 30 gennaio 1937; Scambio di note concernenti la cittadinanza vaticana ai membri delle Rappresentanze della Santa Sede, 23 luglio 1940 - 17 agosto 1940; Scambio di note circa il trasferimento dei privilegi dell'extraterritorialità alle aree cedute in permuta alla Santa Sede, dal Comune di Roma, nella zona di San Paolo, 16 marzo 1945 - 16 aprile 1945; Scambio di Note Verbali fra l'Ambasciata d'Italia e la Segreteria di Stato, per l'esenzione da certe imposte accordata agli agenti diplomatici di nazionalità italiana accreditati presso la Santa Sede da stati terzi, 16 dicembre 1955 (ratifica non necessaria); Scambio di Note Verbali fra la Segreteria di Stato e l'Ambasciata d'Italia relativo alla non applicazione della legge n. 1745 del 29 dicembre 1962 sulla ritenuta di acconto o sugli utili distribuiti dalle società di pertinenza della Santa Sede, 11 ottobre 1963; Scambio di Note Verbali fra la Santa Sede e il Governo Italiano per l'aggiornamento delle liste delle cariche pontificie della Convenzione doganale e della Convenzione sulla circolazione stradale rispettivamente, 24 novembre 1970; Scambio di Note Verbali fra la Segreteria di Stato e l'Ambasciata d'Italia sull'ampliamento del cimitero civile di Albano e il trasferimento al Collegio Urbano di Propaganda Fide a Castel Gandolfo delle immunità diplomatiche di cui agli artt. 15-16 del Trattato Lateranense, 23 gennaio 1981 (ratifica non necessaria); Scambio di Note sulle nomine ecclesiastiche, 23 dicembre 1985; Scambio di Note sulle festività religiose, 23 dicembre 1985; Scambio di note per la costituzione di una Commissione mista come previsto per l'art. 7 del protocollo addizionale del 18 febbraio 1984 che porta le modificazione al concordato lateranense, 13 febbraio 1987; Scambio di Note Verbali fra la Segreteria di Stato e l'Ambasciata d'Italia relativo alla libera cessione al Comune di Castel Gandolfo da parte della Santa Sede di una porzione di area extraterritoriale situata ai margini della Villa Pontificia, 8 agosto 1987 - 7 settembre 1987; Scambio di Note Verbali tra la Segreteria di Stato e l'Ambasciata d'Italia, per l'applicazione al Palazzo San Paolo, in Via della Conciliazione, delle immunità previste dagli artt. 15-16 del Trattato Lateranense, 21 novembre 1989 - 7 settembre 1990; Scambio di Note Verbali fra l'Ambasciata d'Italia e la Segreteria di Stato, circa la doppia cittadinanza dei Cardinali italiani residenti a Roma e del personale di ruolo delle Rappresentanze pontificie, 24 maggio 1990; Scambio di Note Verbali tra l'Ambasciata d'Italia e la Segreteria di Stato relativo al "Passetto di Borgo", 18 maggio 1991; Scambio di note verbali tra l'Ambasciata d'Italia presso la Santa Sede e la Segreteria di Stato concernente il riconoscimento dei titoli accademici conferiti dalle facoltà approvate dalla Santa Sede, 25 gennaio 1994. Scambio di Note con Allegati 1 e 2 tra la Repubblica Italiana e la Santa Sede costituente un'intesa tecnica interpretativa ed esecutiva dell'Accordo modificativo del Concordato Lateranense del 18 febbraio 1984 e del successivo Protocollo del 15 novembre 1984, 10 aprile 1997 e 30 aprile 1997; Scambio di Note, che costituisce un'intesa tecnica esplicativa ed esecutiva, relativo all'applicazione dell'art. 29, comma IV, del Protocollo, firmato il 15 novembre 1984, aggiuntivo all'Accordo del 18 febbraio 1984 tra la Repubblica Italiana e la Santa Sede, 13 novembre 2000 e 14 dicembre 2000; Scambio di Note Verbali tra la Segreteria di Stato e l'Ambasciata d'Italia, per l'applicazione all'immobile di Via della Conciliazione nn. 5/7, delle immunità previste dagli artt. 15-16 del Trattato Lateranense, 18 febbraio 2002 - 16 aprile 2002; Scambio di Lettere fra il Presidente del Consiglio italiano e il Segretario di Stato circa il regime idrico della Città del Vaticano, 2 gennaio 2004 - 13 gennaio 2004; Scambio di Note Verbali tra l'Ambasciata d'Italia e la Segreteria di Stato, circa le modalità di comunicazione delle informazioni di carattere assistenziale e previdenziale dei dipendenti della Santa Sede, degli altri enti centrali della Chiesa gestiti direttamente dalla Santa Sede iscritti in istituti previdenziali italiani, 4 marzo 2004 - 13 marzo 2004; Scambio di Lettere tra il Presidente del Consiglio dei Ministri, Romano Prodi, ed il Cardinale Segretario di Stato di Sua Santità, Angelo Sodano, 26 luglio 2006; Scambio di Note Verbali sul riconoscimento dei titoli accademici 
Si tratta di un procedimento particolare di formazione dei trattati internazionali. E, a riguardo, si ricordi che allorché si manifesti l'esigenza di collaborazione tra Stato e Chiesa per "ulteriori materie", l'art. 13 dell'Accordo del 1984 prevede la possibilità sia di "nuovi accordi tra le due Parti" sia di "intese tra le competenti autorità dello Stato e la Conferenza Episcopale Italiana [C.E.I., d'ora in avanti]"17.

Ratione materiae sono stati siglati in questi anni alcuni "nuovi accordi", che hanno trovato rilevanza nell'ordinamento giuridico italiano attraverso diverse forme di adattamento del diritto interno ${ }^{18}$, ma "per lo più"19 si è provveduto a stipulare intese tra le competenti autorità dello Stato e la Cei, alle quali si è dato esecuzione nel nostro ordinamento attraverso le forme del potere regolamentare ${ }^{20}$ : le così dette intese $d i$ secondo livello, volte a specificare una disciplina di dettaglio, con funzione accessoria, rispetto alle disposizioni di carattere generale contenute nell'Accordo ${ }^{21}$.

pontifici nelle discipline ecclesiastiche, 13 febbraio 2019. Per una rassegna di questi documenti si rimanda alla Banca dati online messa a disposizione dalla piattaforma digitale della Pontificia Università Gregoriana, al seguente link: https://www.iuscangreg. it/accordi_santa_sede.php\#V77.

${ }^{17} \mathrm{La}$ Cei ha acquisito la personalità giuridica civile in forza dell'art. 13 della legge $\mathrm{n}$. 222 del 1985.

18 Ad esempio, all'Intesa per il riconoscimento dei titoli accademici pontifici si è data esecuzione nel diritto interno con il d.p.r. n. 175 del 1994; per l'Accordo tra il Governo italiano e la Santa Sede per regolare i rapporti tra l'ospedale pediatrico "Bambino Gesù" ed il Servizio sanitario nazionale è intervenuta la legge n. 187 del 1995 che ha autorizzato il Presidente della Repubblica alla ratifica; la ratifica della Convenzione di sicurezza sociale tra la Santa Sede e la Repubblica italiana è stata autorizzata con legge n. 244 del 1993; per la Convenzione tra il Governo della Repubblica italiana e la Santa Sede in materia fiscale è intervenuta la legge n. 137 del 2016. L'Accordo concernente l'Ospedale Pediatrico Bambino Gesù, stipulato tramite Scambio di Note diplomatiche il 14 giugno 2018 e il 15 giugno 2018, non ha avuto necessità di essere ratificato in quanto, a fronte dell'esigenza della Santa Sede di adeguare la disciplina normativa dell'Ospedale al diritto vaticano, non muta la posizione giuridica dell'ente nel diritto interno. L'Accordo tra la Santa Sede e la Repubblica Italiana per l'applicazione della Convenzione di Lisbona sul riconoscimento dei titoli di studio relativi all'insegnamento superiore nella Regione Europea, siglato il 13 febbraio 2019, è in attesa di ratifica.

${ }_{19}$ Cfr. E. VITALI, A.G. CHIZZONITI, Manuale breve. Diritto ecclesiastico, 14 ${ }^{\mathrm{a}}$ ed., Giuffrè, Milano, 2019, p. 17.

20 Si pensi ai d.p.r. n. 751 del 1985, n. 202 del 1990 e n. 175 del 2012 sull'insegnamento della religione cattolica; ai d.p.r. n. 571 del 1996, n. 189 del 2000 e n. 78 del 2005 relativi alla tutela dei beni culturali di interesse religioso; ai d.p.r. n. 165 del 1994 e n. 63 del 2019 circa il riconoscimento dei titoli accademici conferite dalle facoltà ecclesiastiche.

${ }^{21}$ Vedi G. CASUSCELLI, Le fonti, in ID. (a cura di), Nozioni di Diritto ecclesiastico, cit., 
Si è portati a ritenere che a quanto previsto dall'art. 11, secondo comma, dell'Accordo del 1984 per le Forze armate si è dato attuazione tramite un "nuovo accordo" di natura internazionalistica. In effetti, anche nello Scambio di lettere diplomatiche, le Parti sostengono che il fine del documento conclusivo approvato dalla Commissione paritetica è quello di "dare attuazione alla previsione dell'articolo 11 di modificazione del Concordato Lateranense"22, proponendo così il testo "dell'Intesa di cui all'Accordo del 1984"23.

Pure i primi commenti dottrinali in materia sembrano concordi nel ritenere che l'Intesa sia "attuativa dell'art. 11 dell'Accordo di revisione del concordato del $1984^{\prime 24}$. La scelta di procedere alla concertazione bilaterale della disciplina attraverso un trattato di natura internazionalistica non sembra, però, essere, a nostro avviso, del tutto coerente con l'impianto concordatario. Infatti, come abbiamo già visto, il richiamato art. 11 fa espresso riferimento a un'intesa fra le autorità italiane competenti e l'autorità ecclesiastica - richiamando, in tal modo, un'intesa di secondo livello - e non un "nuovo accordo" fra le due Parti, la cui possibilità è pure contemplata dall'art. 13, secondo comma, per "ulteriori materie" (e, com'è evidente, l'assistenza religiosa alle Forze armate non è una materia "ulteriore" rispetto a quelle già definite nell'Accordo del 1984). Peraltro, l'interpretazione che ha dato generalmente la dottrina ecclesiasticistica alla previsione contenuta all'art. 11, secondo comma, dell'Accordo del 1984, è stata generalmente quella di un'intesa di derivazione concordataria ${ }^{25}$. Da ciò, si può affermare che, pur perseguendo e attuando lo stesso scopo che si propone l'art. 11, secondo comma, dell'Accordo del 1984, l'Intesa ratificata nell'ordinamento italiano con legge n. 70 del 2021 non è esattamente quell'intesa prevista nel richiamato art. 11.

L'Accordo del 1984 ha potuto rappresentare l'occasione per un'ulteriore negoziazione fra le Parti in materia di assistenza religiosa alle Forze armate, ma non può essere considerato, rispetto all'Intesa, un Accordo-quadro a essa sovraordinato. Con la ratifica nell'ordinamento

p. 37. Sul tema, in generale, si rimanda a P. LILLO, L'adattamento dell'ordinamento interno al "Diritto pattizio". Contributo allo studio delle fonti del diritto ecclesiastico italiano, Giuffrè, Milano, 1992.

${ }^{22}$ Cfr. Gazzetta Ufficiale del 22 maggio 2021, pp. 5 e 8.

${ }^{23}$ Ivi, pp. 6 e 8.

${ }^{24}$ G. DALLA TORRE, Nuova intesa, cit., p. 413.

${ }^{25}$ Sul punto sia consentito rinviare, anche per la bibliografia di riferimento, a L.M. GUZZO, L'assistenza religiosa, cit., pp. 129-136. 
italiano, 1'Intesa ha una sua autonomia, una vita propria rispetto all'Accordo del 1984, avendo acquisito ormai lo stesso valore giuridico sul piano del diritto internazionale.

Inoltre, si è segnato, ancor di più, sotto il profilo ecclesiasticistico, e sotto quello della disciplina specifica che se ne determina, la distanza di trattamento riservato alle Forze armate, rispetto agli altri servizi "assimilabili" alle Forze armate che, pur essendo civili, sono militarmente ordinati26, come la Polizia di Stato, o la polizia penitenziaria ${ }^{27}$.

D'altronde, considerato che l'Intesa interviene a modificare una legge dello Stato - il C.O.M. - si è reso costituzionalmente necessario, ai sensi dell'art. 80 Cost., come già abbiamo precisato, il passaggio parlamentare per autorizzare alla ratifica il Presidente della Repubblica; e ciò spiega come mai detta Intesa non sia stata resa operativa nell'ordinamento dello Stato attraverso un atto avente natura regolamentare ${ }^{28}$. Ciò ha comunque scongiurato che la disciplina finisse nelle more di un sempre più marcato processo di deconcordatarizzazione ${ }^{29}$, a danno delle garanzie di pubblicità e delle prerogative del Parlamento ${ }^{30}$.

\section{3 - La ratifica e l'esecuzione dell'Intesa nell'ordinamento dello Stato, con le relative norme di adeguamento: l'esito paradossale di una disciplina "duplicata"}

Il Capo I della legge in commento contiene sia l'autorizzazione alla ratifica e l'ordine di esecuzione allo Scambio di Lettere (artt. 1 e 2, legge n. 70 del 2021) sia le norme di adeguamento dell'ordinamento interno alle obbligazioni internazionali contratte con la Santa Sede (art. 3, legge n. 70 del 2021).

26 Si parla di "Corpi civili militarmente ordinati" in P.P. RIVELLO, Lezioni di diritto penale militare, 2a ed., Giappichelli, Torino, 2012, p. 19.

27 Cfr. L.M. GUZZO, L'assistenza religiosa, cit., p. XVII ss.

28 Come già abbiamo avuto modo di osservare, l'adeguamento dell'ordinamento a scambi di note diplomatiche può avvenire anche mediante "accordi semplificati" che confluiscono in d.p.r. oppure in decreti ministeriali: vedi G. CASUSCELLI, Le fonti del diritto ecclesiastico italiano: intersezioni e interpretazioni, in S. BERLINGÒ, G. CASUSCELLI, Diritto ecclesiastico italiano. I fondamenti. Legge e religione nell'ordinamento e nella società d'oggi, Giappichelli, Torino, 2020, p. 58.

29 Vedi G. CASUSCELLI, Le fonti, cit., p. 38.

30 Si rimanda a E. VITALI, A.G. CHIZZONITI, Manuale breve, cit., p. 17. 
Di regola, lo Stato può dare attuazione alle obbligazioni contratte in sede internazionale tanto attraverso un procedimento così detto speciale, con un ordine di esecuzione del trattato contenuto in un atto, come una legge di autorizzazione o anche un atto subnormativo, quanto attraverso un procedimento così detto ordinario, con l'emanazione di un atto che riproduce, in maniera identica, il contenuto del trattato ${ }^{31}$.

Nel nostro caso, contestualmente alla ratifica e all'ordine di esecuzione dello Scambio di Lettere, che contiene l'Intesa, sono apportate anche le norme di adeguamento che modificano il C.O.M. Al contrario, per ciò che riguarda le altre obbligazioni contratte con la Santa Sede, alle quali lo Stato italiano si conforma con la legge in esame (l'informazione del pubblico ministero sull'esercizio dell'azione penale nei confronti degli ecclesiastici e il diritto dei cardinali di rendere testimonianza in luogo diverso da quello previsto dalle leggi), non viene data esecuzione ai trattati, predisponendosi, a riguardo, soltanto le norme di adeguamento (artt. 4 e 5 legge n. 70 del 2021).

Ma ritorniamo al Capo I della richiamata legge, alla luce della ratifica e dell'esecuzione dello Scambio di lettere non vi è dubbio che sia stato utilizzato un procedimento "speciale", in cui il trattato, riprodotto in allegato nella lingua autentica (l'italiano), diventa applicabile nell'ordinamento interno. Il legislatore italiano avrà comunque valutato che le norme del trattato non fossero sufficientemente precise nel loro contenuto precettivo, anche con riferimento al "modo di essere dello specifico ordinamento interno" 32 , al punto da ritenere necessaria la predisposizione di talune norme di adeguamento che intervenissero direttamente sul contenuto del diritto militare, modificando alcuni articoli del Codice.

L'esito è, però, quasi paradossale, soprattutto allorché si osservi come, in realtà, le norme dell'Intesa trovano operatività immediata nell'ordinamento italiano. Infatti, già a partire dalla loro formulazione, le norme dell'Intesa appaiono abbastanza chiare e precettive nel contenuto; da questo punto di vista, non si comprende appieno la scelta del legislatore italiano di produrre delle norme di adeguamento, con un intervento, quasi certosino, di "taglia e cuci" all'interno del Codice. Potrebbe dirsi che, in tal modo, si sono voluti preservare i principi dell'unità, della sistematicità e della coerenza, sulle cui basi teoriche e

31 Sul tema la letteratura è abbondante ma si rinvia, per tutti, a C. FOCARELLI, Diritto internazionale, 3a ed., Wolters Kluwer-Cedam, Milano-Padova, 2015, p. 209 ss.

${ }^{32}$ Cfr., sul punto, C. FOCARELLI, Diritto internazionale, cit., p. 211. 
storiche si è costruito anche il recente e ultimo processo di codificazione militare italiana.

Il contenuto del trattato internazionale risulta incorporato doppiamente nel nostro ordinamento giuridico. Più correttamente, l'Intesa assume la qualità di una fonte di produzione per determinate norme che, concertate bilateralmente, sono inserite all'interno di una legge dello Stato, il C.O.M. La conseguenza è che nel C.O.M., una fonte legislativa di rango ordinario, sono inserite delle norme che hanno una particolare forza passiva di resistenza all'abrogazione; delle norme, cioè, "rinforzate" rispetto alle altre norme ordinarie.

L'Intesa, nei fatti, non sostituisce l'intero titolo III del libro V del C.O.M., relativo alla disciplina del Servizio di assistenza spirituale cattolico, ma modifica e abroga alcuni articoli, lasciando immutate, nella loro formulazione, altre disposizioni. Ne deriva che uno stesso istituto l'assistenza religiosa alle Forze armate per i cattolici - è ora disciplinato sia da norme ordinarie sia da norme con una particolare resistenza passiva. Ciò non tanto - com'è noto - per una sorta di "costituzionalizzazione" che, a norma dell'art. 7 Cost., arriverebbe ad assorbire finanche le disposizioni attuative dell'Accordo del 1984, quanto in virtù dell'art. 10 Cost. e dell'art. 117, terzo comma, Cost. ${ }^{33}$.

A ciò si aggiunga una differenza di natura temporale nell'entrata in vigore delle due fonti. Infatti, le disposizioni che novellano il C.O.M. sono entrate in vigore, ai sensi dell'art. 7 della legge in commento, il giorno successivo a quello della pubblicazione nella Gazzetta Ufficiale, ossia il 23 maggio 2021. Ma per l'entrata in vigore dell'Intesa, ai sensi del suo art. 14, non è stata sufficiente la pubblicazione nella Gazzetta Ufficiale, essendo necessaria anche la pubblicazione negli Acta Apostolicae Sedes, avvenuta in un numero speciale del 15 settembre 2021 (nonostante la previsione della contestualità della pubblicazione nelle due raccolte ufficiali - "in pari data" si legge nella disposizione - non sia stata rispettata). Insomma, le norme di adeguamento sono entrate in vigore prima dell'Intesa. Ciò non toglie, comunque sia, che le norme di adeguamento fossero già pienamente operative dall'entrata in vigore della legge, come vedremo più approfonditamente nel par. 8 .

${ }^{33}$ La bibliografia sul punto è ampia e il dibattito dottrinale registra diverse posizioni in materia, anche con riferimento alla giurisprudenza costituzionale. Si rimanda, per tutti, a F. FINOCCHIARO, Diritto ecclesiastico, 12a ed., aggiornamento a cura di A. BETTETINI e G. LO CASTRO, Zanichelli, Milano, 2015, pp. 126-130. 


\section{4 - Le ondivaghe "vicissitudini" dell'art. 17 C.O.M.: un "termostato" delle tensioni sociali e giuridiche riguardanti la materia e l'esigenza di una riforma}

Il problema principale, alla luce del principio supremo di laicità dell'ordinamento repubblicano e degli obblighi assunti in sede concordataria, risiede, a nostro avviso, proprio nella regolamentazione dettagliata dell'assistenza religiosa solo per i cattolici all'interno di una legge unilaterale dello Stato.

Ciò è plasticamente dimostrato dalle "vicende" (rectius: "vicissitudini", è il caso di dire) caratterizzanti l'art. 17 C.O.M., che in poco più di dieci anni (undici, per la precisione) ha avuto a oggi ben tre formulazioni (con buona pace di un ulteriore valore cardine delle codificazioni moderne e contemporanee: la certezza del dritto!).

Nella versione originaria - così come compare nel testo del d.lgs.vo n. 66 del 2010 pubblicato sulla G.U. dell'8 maggio 2010 - 1'art. 17 C.O.M. disponeva che:

"Il Servizio di assistenza spirituale alle Forze armate, istituito per assicurare l'esercizio delle pratiche di culto del personale militare di religione cattolica e disimpegnato da sacerdoti cattolici in qualità di cappellani militari, è disciplinato dal titolo III del libro V".

La disposizione restringeva, sotto il profilo teleologico, gli scopi dell'assistenza religiosa ai militari cattolici al solo "esercizio delle pratiche di culto" circoscrivendone (e di molto) le finalità rispetto a quanto prevedeva la normativa previgente, cioè l'art. 1 della legge n. 512 del 1961, a norma della quale il servizio dell'assistenza religiosa veniva istituito "per integrare secondo i principi della religione cattolica, la formazione spirituale delle Forze Armate stesse". Il riferimento alle sole pratiche di culto si presentava agli occhi della dottrina come una "formulazione più sobria e rispondente al principio di laicità dello Stato"34. Ma la perentorietà della norma che riconduceva l'assistenza religiosa alla sola legislazione unilaterale dello Stato appariva comunque in evidente contraddizione con quanto previsto dall'art. 11, secondo comma, dell'Accordo del 1984 che, al contrario, come già si è visto, prescriveva una concertazione bilaterale della regolamentazione del servizio.

${ }^{34}$ Così G. DALLA TORRE, Lezioni di Diritto ecclesiastico, 5ª ed., Giappichelli, Torino, 2014, p. 323. 
Il d.lgs.vo n. 20 del 24 febbraio 2012, apportante modifiche e integrazioni al Codice dell'ordinamento militare, all'art. 1, lett. b) riformulava quindi l'art. 17, primo comma, C.O.M. nei seguenti termini:

"Il Servizio di assistenza spirituale alle Forze armate, istituito per
integrare la formazione spirituale del personale militare di religione
cattolica e disimpegnato da sacerdoti cattolici in qualità di cappellani
militari, fino all'entrata in vigore dell'intesa prevista all'articolo 11,
secondo comma, dell'Accordo, con protocollo addizionale, firmato a
Roma il 18 febbraio 1984, che apporta modificazioni al Concordato
Lateranense dell'11 febbraio 1929, tra la Repubblica italiana e la Santa
Sede, ratificato e reso esecutivo con la legge 25 marzo 1985, n. 121, è
disciplinato dal presente codice e, in particolare, dal titolo III del libro
$V^{\prime \prime} 35$.

In tal modo, l'atteggiamento del legislatore italiano appariva quasi "a fisarmonica", un passo indietro e uno in avanti: mentre le finalità dell'assistenza religiosa ai militari cattolici ritornavano come volte a "integrare la formazione spirituale del personale militare di religione cattolica", si concepiva (o, forse, sarebbe meglio dire: si tollerava) come provvisoria la presenza di una disciplina giuridica riservata ai militari cattolici, al pari di quanto dimostra l'utilizzo della locuzione prepositiva "fino a" nel testo della norma. Pareva presupporsi, insomma, che il titolo III del libro V del Codice fosse destinato a essere abrogato una volta entrata in vigore l'Intesa prevista dall'Accordo del $1984^{36}$. Ciò poteva intendersi pure da una prima lettura dell'art. 14, secondo comma, della stessa Intesa:

"Con l'entrata in vigore delle presenti norme cessano di avere efficacia le disposizioni di cui al titolo III, Libro V, del Codice dell'Ordinamento militare e tutte le altre disposizioni legislative e regolamentari con esse incompatibili".

Nei fatti, però, avendo il legislatore italiano optato per la produzione di norme di adeguamento che novellano il titolo III, Libro V del C.O.M., il richiamato titolo III non è stato formalmente abrogato. D'altronde, l'Intesa non interviene su alcuni aspetti che pure sono

\footnotetext{
${ }^{35}$ Mio il corsivo

${ }^{36}$ Come sostenuto anche nella dottrina, "il servizio di assistenza religiosa per i cattolici nell'ambito delle forze armate è disciplinato al momento in via unilaterale dal d.lgs. n. 66 del 2010 (cd. Codice dell'ordinamento militare) in attesa che sia stipulata l'intesa prevista dall'art. 12 dell'Accordo del 1984 (art. 17)", A. VALSECCHI, L'assistenza spirituale, cit., p. 210 .
} 
disciplinati all'interno del titolo III (ad esempio, le religiose in servizio presso gli stabilimenti sanitari militari; sul punto, torneremo nel successivo par. 9), e ciò ha reso problematico provvedere a un'abrogazione formale dell'intero titolo III. Si è così resa necessaria una nuova versione dell'art. 17 C.O.M. , introdotta dall'art. 3.1, lett. a), della legge in commento:

"1. L'assistenza spirituale ai militari cattolici, di cui all'articolo 11, secondo comma, dell'accordo, con protocollo addizionale, firmato a Roma il 18 febbraio 1984, che apporta modificazioni al Concordato lateranense dell'11 febbraio 1929, tra la Repubblica italiana e la Santa Sede, ratificato e reso esecutivo con la legge 25 marzo 1985, n. 121, è assicurata da cappellani militari, nominati dal Ministro della difesa su designazione dell'Ordinario militare, in base alle disposizioni stabilite dal presente codice e, in particolare, dal titolo III del libro quinto.

2. Le autorità militari garantiscono ai cappellani militari la piena libertà nell'esercizio del loro ministero, riconoscendo la dignità e la natura peculiare del loro servizio, e assicurano la disponibilità dei luoghi e dei mezzi necessari per l'assolvimento delle loro funzioni".

Come si vede, quindi, l'art. 17 C.O.M. nella formulazione vigente si limita a richiamare l'art. 11, secondo comma, dell'Accordo del 1984, senza menzionare l'Intesa raggiunta il 13 febbraio 2018. Questa scelta legislativa non risulta del tutto corretta, in quanto, come già si è detto, la fonte di produzione delle norme di adeguamento, il cui contenuto è stato concertato bilateralmente, non è l'art. 11, secondo comma, dell'Accordo del 1984, bensì il "nuovo accordo" di natura internazionalistica, dalle Parti qualificato come Intesa.

Inoltre, la nuova formulazione della disposizione mette in evidenza come i soggetti attuatori dell'assistenza spirituale siano i cappellani militari nominati secondo le norme del titolo III del libro V del Codice. Non si fa più riferimento alle finalità dell'assistenza spirituale, che saranno comunque riprese nel nuovo art. 1533-bis C.O.M., con cui si richiama la soddisfazione delle "esigenze spirituali del personale [...] e dei relativi familiari", che viene inserito nell'art. 17 C.O.M., secondo comma, relativo alla libertà nell'esercizio del ministero riconosciuta ai cappellani militari.

L'art. 14, secondo comma, dell'Intesa, che assume la funzione di una norma di chiusura, deve, quindi, essere interpretato come norma che abroga non tutte le disposizioni del titolo III del Codice ma soltanto quelle "incompatibili" con l'Intesa (la clausola presente nella norma "con esse incompatibili" si riferisce tanto alle norme del titolo III quanto alle altre disposizioni legislative e regolamentari). 
Questa breve analisi di tipo quasi "filologico", dell'art. 17 C.O.M. ci porta ad affermare che le differenti formulazioni assunte dalla disposizione nel corso di pochi anni rappresentano un po' il "termostato" delle tensioni sociali e giuridiche che hanno riguardato e riguardano la materia nei tentativi di riforma. Esse, infatti, danno conto delle tappe indeterminate, delle esitazioni e dei tentennamenti dovute, a nostro avviso, al tentativo di giustificare un istituto dalle marcate tendenze giurisdizionaliste, come l'inserimento organico dei cappellani nelle strutture militari, che mal si concilia con il contesto legale di un ordinamento giuridico repubblicano che annovera la laicità quale principio supremo ${ }^{37}$.

\section{5 - Politica concordataria della Santa Sede e attribuzioni alle Conferenze episcopali nazionali. Alcune considerazioni sulla polarizzazione del dibattito pubblico in Italia}

Prima di passare in rassegna il contenuto dell'Intesa e delle relative norme di adeguamento ci soffermiamo ancora sulle modalità di concertazione bilaterale che intersecano altresì altre questioni che vanno polarizzandosi nel dibattito pubblico italiano.

Nonostante le considerazioni che abbiamo svolto, la scelta di intraprendere la strada di una modificazione di legge attraverso un trattato appartiene all'ambito discrezionale che connatura la sovranità degli Stati come soggetti di diritto internazionale. La Santa Sede, d'altra parte, per le ragioni storiche a tutti note, al pari degli Stati sovrani e insieme agli Stati, negozia obbligazioni internazionali su materie inerenti all'ordine spirituale ${ }^{38}$. In tal senso, la Chiesa cattolica si conferma come "un unicum sia fra gli Stati che fra le religioni"39. Inoltre, vi è da aggiungere che sulla materia dell'assistenza religiosa alle Forze armate la Santa Sede è già intervenuta in diverse occasioni, tramite accordi

\footnotetext{
37 Come ha sottolineato Consorti, la materia è espressione di "un certo collateralismo fra Stato e Chiesa cattolica, a sua volta manifestazione dell'omogeneità culturale della società italiana": P. CONSORTI, Diritto e religione. Basi e prospettive, Laterza, Roma-Bari, 2020, p. 185.

${ }^{38}$ La bibliografia sul tema è molto ampia. Si rimanda per tutti a S. FERLITO, L'attività internazionale della Santa Sede, Giuffrè, Milano, 1988.

${ }^{39}$ Così P. CONSORTI, Le riforme economiche di Papa Francesco, in E. BANI, P. CONSORTI (a cura di), Finanze vaticane e Unione europea. Le riforme di Papa Francesco e le sfide della vigilanza internazionale, il Mulino, Bologna, 2015, p. 9.
} 
internazionali, concernenti di solito pure le Forze di polizia ${ }^{40}$. In Italia, al contrario, come già si è detto, l'assistenza religiosa alla Polizia di Stato è stata regolamentata tramite una fonte di secondo livello, mentre per quella alle Forze armate si è provveduto con un nuovo accordo di natura internazionalistica. D'altra parte, un accordo di tale natura non si rendeva nei fatti necessario, in quanto rimaneva sufficiente la copertura offerta dall'art. 11 dell'Accordo 1984, rispetto al quale semmai poteva darsi attuazione con un atto subconcordatario e di dettaglio.

Quello che ora interessa porre in evidenza è che, a livello di rapporti intraecclesiali, la scelta di perseguire la strada di un accordo internazionalistico ha comportato l'esclusione della Conferenza Episcopale Italiana (C.E.I.) dal tavolo di concertazione della disciplina normativa dell'assistenza religiosa per i militari cattolici in Italia. Vi sarebbe da chiedersi, in effetti, quanto l'accentramento sulla Santa Sede della politica ecclesiastica nel diritto internazionale finisca per frustrare le competenze delle conferenze episcopali nazionali, soprattutto dinnanzi a un magistero di papa Francesco che pare andare, invece, nella direzione di una maggiore valorizzazione delle assemblee nazionali di vescovi in chiave sinodale ${ }^{41}$.

${ }^{40}$ Cfr. l'Acuerdo entra la República Argentina y la Santa Sede sobre jurisdicción Castrense y Asistencia religiosa de las Fuerzas Armadas, firmato il 18 giugno 1957 e reso esecutivo l'8 luglio 1957; 1'Acuerdo entra la Santa Sede y la República de Bolivia sobre asistencia religiosa a las Fuerzas Armadas y Fuerzas de la Policía Nacional, firmato l'1 dicembre 1986 e reso esecutivo il 2 marzo 1989; l'Acuerdo entre la Santa Sede y la República del Ecuador sobre asistencia religiosa a las Fuerzas armadas y Policía nacional, firmato il 3 agosto 1978 e reso esecutivo il 26 marzo 1983 (con Scambio di Note diplomatiche esplicative nel 1982); il Convenio entre la Santa Sede y la Republica de El Salvador sobre jurisdicción eclesiástica castrense y asistencia religiosa de la Fuerza Armada y Cuerpos de Seguridad, firmato l'11 marzo 1968 e reso esecutivo il 2 luglio 1968; il Convenio entre la Santa Sede y la República del Paraguay sobre asistencia religiosa a las Fuerzas Armadas de la Nación y la Policía Nacional, firmato il 24 dicembre 2002 e reso esecutivo il 18 ottobre 2004; 1'Accordo tra la Santa Sede e la Bosnia ed Erzegovina circa l'assistenza religiosa ai fedeli cattolici, membri delle Forze Armate di Bosnia ed Erzegovina, firmato 1'8 aprile 2010 e reso esecutivo il 14 settembre 2010; 1'Accordo tra la Santa Sede e la Repubblica di Croazia circa l'assistenza religiosa ai fedeli cattolici, membri delle forze armate e della polizia della Repubblica di Croazia, firmato il 19 dicembre 1996 e reso esecutivo il 9 aprile 1997; l'Accordo tra la Santa Sede e la Repubblica Slovacca circa l'assistenza religiosa ai fedeli cattolici nelle Forze Armate e nei Corpi Armati della Repubblica Slovacca, firmato il 21 agosto 2002 e reso esecutivo il 28 ottobre 2002; 1'Accordo tra la Santa Sede e lo Stato Spagnolo circa l'assistenza religiosa alle forze armate ed il servizio militare degli ecclesiastici e religiosi, firmato il 3 gennaio 1979 e reso esecutivo il 4 dicembre 1979. Sul tema cfr. anche C. CORRAL SALVADOR, Derecho internacional concordatario, Biblioteca de Autores Cristianos, Madrid, 2009, p. 236 ss.

${ }^{41}$ Ciò vale anche, ad esempio, in materia liturgica, con la modifica del can. 838 c.i.c.: 
Con riguardo al caso specifico, osserviamo che gli ordinariati militari sono circoscrizioni assimilabili a una diocesi ${ }^{42}$. A ragione di ciò, l'Ordinario militare per l'Italia fa parte di diritto della C.E.I. ${ }^{43}$. Ma in circostanze come questa nonché in altre circostanze, l'Ordinariato militare sembra più essere, sul piano sostanziale, una prelatura personale alle dirette dipendenze della Santa Sede.

Un maggior coinvolgimento delle Conferenze nazionali e, nello specifico, della Conferenza episcopale italiana per gli affari che riguardano l'Ordinariato militare sarebbe stato auspicabile, non soltanto in questo caso $^{44}$. Non si comprendono appieno le ragioni, in termini strettamente

FRANCESCO, Lettera apostolica in forma di Motu Proprio "Magnum Principium", Città del Vaticano, 3 settembre 2017 (in https://press.vatican.va/content/salastampa/it/bollettino /pubblico/2017/09/09/0574/01279.html\#la). D'altronde, le Conferenze episcopali nazionali hanno acquisito un ruolo importante, quasi come "cerniera" nel rapporto tra le Chiese particolari e la Chiesa universale, nel processo sinodale che coinvolge la Chiesa dal 2021 al 2023, con la XVI Assemblea Generale Ordinaria del Sinodo dei Vescovi. Cfr. la Nota del Sinodo dei vescovi, 21 maggio 2021, in cui si dà conto del "nuovo itinerario sinodale" approvato da Papa Francesco in data 24 aprile 2021-

${ }^{42}$ Cfr. GIOVANNI PAOLO II, Costituzione Apostolica Spirituali Militum Curae, par. 1, Città del Vaticano, 24 aprile 1986 (in http://www.ordinariatomilitare.it/diocesi/legislazione/ canonica/documento-del-papa/). Cfr. F. VECCHI, voce Ordinario militare, in Enciclopedia Giuridica, Aggiornamento XV, Istituto della Enciclopedia Italia fondata da Giovanni Treccani, Roma, 2007, p. 2006 ss.

43 Cfr. GIOVANNI PAOLO II, Costituzione Apostolica Spirituali Militum Curae, cit., par. 4, n. III. Vedi anche l'art. 10 degli Statuti dell'Ordinariato militare in Italia, approvati dalla Santa Sede in data 6 agosto 1987 (si possono trovare al seguente link: http://www. ordinariatomilitare.it/diocesi/legislazione/canonica/statuti/).

${ }_{44}$ Non è la prima volta che la C.E.I. viene surclassata in questioni che riguardano l'Ordinariato militare. Ci si ricorderà, ad esempio, del decreto del 17 giugno 2017 con il quale la Congregazione per il Culto divino e i Sacramenti confermava la scelta dell'Ordinariato militare di eleggere San Giovanni XXIII a Patrono dell'Esercito italiano (il provvedimento può trovarsi al seguente link: http://www.esercito.difesa.it/storia/ Documents/Roncalli/decreto-latino.pdf), senza neanche informare la C.E.I., tanto che il presidente della C.E.I. card. Gualtiero Bassetti, ai giornalisti che gli chiedevano conto della decisione adottata dal dicastero della Curia romana, si era sentito in dovere di rispondere che: "è una questione su cui non voglio entrare perché purtroppo ne sono stato informato questa mattina". Si veda la cronaca riportata su Avvenire, il quotidiano dei vescovi italiani: E. LENZI, L'annuncio. San Giovanni XXIII patrono dell'esercito tra tanti dubbi, in Avvenire.it, 12 settembre 2017 (https://www.avvenire.it/chiesa/pagine/san-giovannixxiii-sar-patrono-dellesercito-italiano). La "mattina" di cui parla il porporato nella dichiarazione si riferisce al giorno della cerimonia presso il Palazzo dell'Esercito, in cui l'Ordinario militare ha consegnato al Capo di Stato Maggiore dell'Esercito il decreto emanato dalla Congregazione vaticana: il 12 settembre 2017, ben tre mesi dopo la data di promulgazione dell'atto. Sono stati sollevati diversi dubbi circa la legittimità della 
pratici e operativi, per le quali la Conferenza episcopale italiana può legittimamente intervenire quando si tratta di assistenza religiosa alla Polizia di Stato (così come nel caso dell'insegnamento della religione cattolica nelle scuole pubbliche ${ }^{45}$ ), ma viene del tutto estromessa per la materia inerente all'assistenza religiosa alle Forze armate.

È opportuno ricordare, a riguardo, che la giurisdizione dell'Ordinario militare è

"propria ma cumulativa con la giurisdizione del vescovo diocesano, poiché le persone appartenenti all'Ordinariato non cessano di essere fedeli di quella Chiesa particolare del cui popolo, in ragione del domicilio o del rito, costituiscono una porzione" 46 .

Da qui, si comprende facilmente l'esigenza, anche pastorale, di una più stretta collaborazione dell'Ordinario militare con gli altri vescovi che operano nello stesso territorio nazionale.

In Italia, insomma, la C.E.I. non è stata coinvolta nel processo di revisione del servizio di assistenza religiosa per i cattolici nelle Forze armate. E ciò ha conseguenze importanti sul piano del dibattito pubblico. Infatti, la necessità di una revisione dell'istituto è stata ed è al centro di una discussione che ha coinvolto e coinvolge ampi strati della società civile.

Il tema, com'è ovvio, riguarda un certo "modo" di intendere la religione e i suoi rapporti con i poteri statali, nonché le relazioni tra la Chiesa cattolica e il mondo militare, come già aveva dimostrato anche la riflessione di don Lorenzo Milani ${ }^{47}$ e la vicenda giudiziaria che ne è conseguita ${ }^{48}$.

procedura canonica seguita per l'elezione di San Giovanni XXIII a Patrono dell'Esercito italiano. Cfr. P. CONSORTI, San Giovanni XXIII papa: patrono efficace dell'Esercito italiano?, in Settimana News, 19 settembre 2017; ID., L'oblio dell'ortoprassi e il trionfo dell'ortodossia, blog dell'Università di Pisa, 7 ottobre 2017 (https://people.unipi.it/pierluigi_consorti/lobliodellortoprassi-e-il-trionfo-dellortodossia/).

${ }^{45} \mathrm{Cfr}$. Intesa per l'insegnamento della religione cattolica nelle scuole pubbliche tra il Ministro dell'Istruzione, dell'Università e della Ricerca e il Presidente della Conferenza Episcopale Italiana, 28 giugno 2012, che apporta modifiche all'Intesa stipulata il 14 dicembre 1985, successivamente modificata il 13 giugno 1990.

${ }^{46}$ GIOVANNI PAOLO II, Costituzione Apostolica Spirituali Militum Curae, cit., par. 4, n. IV.

47 La riflessione "milaniana" è una critica aperta al militarismo come "idea istituzionalizzata per esercitare violenza contro i fratelli, per alimentare le guerre anziché contribuire alla costruzione di una società pacifica", secondo G. GUZZO, Don Lorenzo Milani. Un rivoluzionario, un santo, un profeta o un uomo?, Rubbettino, Soveria Mannelli, 1998, p. 105. Si veda anche, dello stesso autore, G. GUZZO, Don Lorenzo Milani. Un 
La discussione si inserisce, altresì, nelle dinamiche di funzionamento della Chiesa cattolica e sul processo di riforma che porta avanti il pontificato di Francesco. Ma la stessa discussione è stata poi fagocitata nel vortice di chi tende a semplificare e a polarizzare il dibattito sul pontificato di Francesco in ampie fazioni, in "tendenze antropologiche ed etiche" 49 , sintetizzabili tra favorevoli e contrari ${ }^{50}$. Secondo Alex Zanotelli, direttore di "Mosaico di Pace", la posizione di Papa Francesco sarebbe abbastanza chiara: "per assistere spiritualmente i soldati, non servono sacerdoti con i gradi". Sulla base di una simile interpretazione, il religioso comboniano si appella direttamente a Papa Bergoglio per rendere "più credibile la scelta della Chiesa per la pace"51. Anche secondo il Consiglio Nazionale Comunità di Accoglienza (CNCA) la conclusione dell'azione pastorale di Francesco - il quale è andato a pregare sulla tomba di don Milani, a Barbiana - sarebbe scontata: "portar fuori dalla struttura militare il ruolo e l'azione dei preti impegnati per l'accompagnamento pastorale di chi presta servizio militare"52. Il Sindacato Autonomo dei Finanzieri, in un editoriale fortemente critico nei confronti dell'Intesa raggiunta, ha tirato in ballo la stessa C.E.I., domandandosi in maniera quasi provocatoria: "ma chi ha voluto questa intesa? La Conferenza Episcopale Italiana o forse 1'Ordinariato militare?"53, per poi proseguire:

"il ruolo dei Cappellani militari è in contraddizione con le stesse parole e azioni del messaggio di pace e fraternità rivelato da Gesù nei

itinerario pedagogico, Rubbettino, Soveria Mannelli, 1988.

48 Cfr. L. MILANI, Lettera ai cappellani militari. Lettera ai giudici, a cura di S. TANZARELLA, Il Pozzo di Giacobbe, Trapani, 2017.

${ }^{49}$ R. D'AMBROSIO, Ce la farà Francesco? La sfida delle riforme ecclesiale, La Meridiana, Molfetta (BA), 2016, p. 12.

50 Cfr. A. MANTINEO, Il Papa venuto dalla "fine del mondo" per la Chiesa del terzo millennio, in A. MANTINEO, L.M. GuZZO (a cura di), Il Papa venuto dalla "fine del mondo" per la Chiesa del terzo millennio, Wolters Kluwer-Cedam, Milano - Padova, 2019, p. 8 ss.

51 A. ZANOTELLI, Cappellani militari: mi appello a Papa Francesco, in Pressenza. International Press Agency, 13 marzo 2018 (https://www.pressenza.com/it/2018/03/cappellanimilitari-mi-appello-papa-francesco/).

${ }^{52} \mathrm{Cfr}$. Lettera del CNCA alla CEI, Smilitarizzare i cappellani militari per prendere sul serio la lezione di don Milani, 17 giugno 2017 (in https://www.cnca.it/smilitarizzare-i-cappellanimilitari-per-prendere-sul-serio-la-lezione-di-don-milani/).

53 Così F. PERROTTA, Libertà religiosa e laicità nelle Amministrazioni militari, in Sindacato Autonomo dei Finanzieri, 30 marzo 2021, p. 5. 
Vangeli e, anche, con il magistero di Papa Francesco contro la guerra e a favore della non violenza" 54 .

Certamente, la presenza organica di sacerdoti all'interno dei ruoli militari non è facilmente conciliabile con la netta condanna di Papa Francesco rivolta alla guerra e alle politiche che distolgono le risorse dalla società civile per orientarli agli sforzi militari:

"la guerra, che orienta le risorse all'acquisto di armi e allo sforzo militare, distogliendole dalle funzioni vitali di una società, quali il sostegno alle famiglie, alla sanità e all'istruzione, è contraria alla ragione, secondo l'insegnamento di san Giovanni XXIII"55.

Ma nei fatti non è, comunque, semplice rintracciare nel magistero del primo Pontefice latino-americano un'affermazione netta sulla necessità di smilitarizzare i cappellani militari ${ }^{56}$. Papa Francesco sui gradi militari per i cappellani non si è mai pronunciato in maniera diretta ${ }^{57}$. Da qui, in dottrina è stato sottolineato che il messaggio di pace di Francesco è "molto incentrato sul contrasto reale alla povertà e impegnato a negare a livello dottrinale il concetto di guerra giusta [...] piuttosto che a fornire supporto alla logica del disarmo a tutti i costi" 58 .

Colpisce, quindi, la non corretta narrazione che si è creata sul tema nell'opinione pubblica. Le istanze di smilitarizzazione dei cappellani militari sono state direttamente ricondotte al Romano Pontefice, polarizzando il tema in una supposta contrapposizione tra Papa Francesco, da un lato, ed episcopato italiano e Ordinariato militare,

${ }^{54}$ F. PERROTTA, Libertà religiosa, cit.

55 FRANCESCO, Incontro con $i$ vescovi del Mediterraneo. Discorso del Santo Padre Francesco, Bari, 23 febbraio 2020 (in http://www.vatican.va/content/francesco/it/speeches/2020/ february/documents/papa-francesco_20200223_visita-bari.html).

56 A differenza dell'arcivescovo Ordinario militare per l'Italia mons. Santo Marcianò, il quale in un'intervista rilasciata a un noto programma televisivo, pochi giorni dopo essere stato eletto pastore della Chiesa castrense in Italia - il 10 ottobre 2013 - aveva affermato: "noi preti non siamo fatti né per i soldi né per i gradi" (cfr. L.M. GUZZO, Verso la riforma, cit., p. 1), e l'anno successivo su "Avvenire", il quotidiano dei vescovi italiani, aveva dichiarato: "lo stato giuridico dei cappellani militari non è legato né allo stipendio né tanto meno ai gradi" (cfr. l'intervista rilasciata a G. CARDINALE, Marcianò: in caserma una Chiesa impegnata al servizio della pace, in Avvenire, 15 aprile 2014, p. 18).

$57 \mathrm{D}$ 'altronde, considerato che il loro statuto giuridico è diversamente modulato dai diritti concordatari nonché dalle singole discipline nazionali e che l'integrazione organica rappresenta soltanto uno dei modelli di strutturazione dei rapporti fra gli stati e le chiese in materia, un intervento diretto del pastore della Chiesa universale potrebbe interferire eccessivamente con le attribuzioni delle conferenze nazionali e degli episcopati locali.

58 A. DE OTO, Altare e mostrine, cit., p. 10. 
dall'altro. Ma l'intervento diretto della Segreteria di Stato evidenzia i limiti strutturali di una simile lettura, in quanto l'accordo in questione rientra nella politica concordataria attuata dalla Santa Sede sotto il pontificato di Francesco. Si potrebbe semmai parlare delle resistenze che l'azione pastorale di Papa Francesco incontra nella Curia romana ${ }^{59}$, a causa del "suo ostinato ed insieme coraggioso ritorno al Concilio Vaticano II, per darne piena ed autentica attuazione" 60 . Ma tale questione esula dalle finalità e dall'oggetto saggio.

\section{6 - I contenuti dell'Intesa}

Passiamo ora ad analizzare i contenuti dell'Intesa. Gli artt. 1 e 2 della legge n. 70 del 2021 riguardano l'autorizzazione alla ratifica e l'ordine di esecuzione dello Scambio di Lettere.

L'Intesa consta di quattordici articoli. Gli artt. 1 e 2 individuano le funzioni svolte dai cappellani per i militari cattolici e i rispettivi familiari, nonché i mezzi e gli strumenti a loro disposizione.

L'art. 3 prevede la possibilità per i cappellani di avvalersi nelle attività di culto di altri sacerdoti in servizio presso la diocesi competente per territorio, salvo comunicazione alle autorità militari competenti da parte dell'Ordinario militare e, comunque, senza oneri aggiuntivi per l'Amministrazione. Il secondo comma della disposizione prevede, inoltre, che in caso di assenza il cappellano è sostituito dal parroco "competente per la sede di servizio", previa comunicazione dell'Ordinario militare al Comandante della sede e senza oneri aggiuntivi per l'Amministrazione.

A norma dell'art. 4 la "direzione e il coordinamento" del servizio di assistenza spirituale spettano ${ }^{61}$ all'Ordinario militare, nominato dal Presidente della Repubblica su designazione della Santa Sede. Il Vicario generale, secondo gli alinea n. 2 e n. 3 della stessa disposizione, è nominato dal Presidente della Repubblica su designazione dell'Ordinario militare; coadiuva quest'ultimo e lo sostituisce in caso di "sede vacante o

59 Vedi, per tutti, M. POLITI, Francesco tra i lupi. Il segreto di una rivoluzione, Laterza, Roma-Bari, 2015; M. MARZANO, La Chiesa immobile. Francesco e la rivoluzione mancata, Laterza, Roma-Bari, 2018.

${ }^{60}$ A. MANTINEO, Il Papa venuto dalla "fine del mondo", cit., p. 5.

${ }^{61} \mathrm{Nel}$ testo dell'Intesa è usato il singolare "spetta" come se - al di là della questione grammaticale - "direzione" e "coordinamento" esprimessero il medesimo concetto. Nel correlato adeguamento del C.O.M. alla lettera del novellato art. 1533 compare in modo corretto il plurale "spettano". 
di impedimento", rappresentandolo "quando non può personalmente intervenire". Il secondo comma stabilisce che l'Ordinario militare può "avvalersi di cinque Cappellani coordinatori presso gli Stati maggiori di Forza armate e i Comandi generali", i cui nomi sono comunicati dall'Ordinario al Ministro della difesa. Il terzo comma si riferisce all'individuazione delle sedi in cui è prestata l'assistenza spirituale, determinate con "apposito decreto" dal Ministro della difesa, sentito l'Ordinario militare, il quale dovrà individuare la sede per ciascun cappellano, comunicandola alla competente Autorità militare. Il quarto comma precisa che le funzioni esercitate dall'Ordinario militare nei riguardi dei cappellani, del personale e dei loro familiari sono "funzioni episcopali".

L'art. 5 definisce l'organico e lo stato giuridico dei cappellani. Al secondo comma è stabilito l'attribuzione dei gradi militari "per assimilazione" con l'intento di "garantire che al Cappellano sia riconosciuta la dignità delle sue funzioni, e per consentire una piena agibilità delle strutture militari allo scopo di assolvere il servizio ministeriale". Il successivo art. 6 definisce e determina l'assimilazione ai gradi.

Nell'art. 7 sono individuate le modalità e le forme di avanzamento stabilendo che le promozioni dei cappellani si effettuano per "anzianità congiunta al merito" [dal grado di sottotenente di completamento al grado di tenente in servizio permanente effettivo (spe), e dal grado di tenente spe al grado di capitano spe] e per "merito comparativo" (dal grado di capitano al grado di maggiore spe). Sono stabiliti anche i periodi di permanenza minima per la promozione (secondo comma) e l'indicazione delle valutazioni di merito sulla "capacità e l'idoneità dei soggetti interessati" (terzo comma).

L'art. 8 riguarda il rapporto di impiego. La nomina a cappellano militare è "disposta su designazione dell'Ordinario militare" e il cappellano deve godere dei diritti civili e politici, avere l'idoneità incondizionata al servizio militare, non avere meno di 28 anni e più di 40 anni. Secondo quanto prevede il secondo comma il rapporto di impiego consiste "nell'esercizio del ministero sacerdotale".

L'art. 9 definisce il trattamento economico che, a norma del primo comma, è quello "di base previsto per gli ufficiali delle Forze armate secondo il grado di assimilazione, con la relativa progressione economica". Il secondo comma della disposizione riguarda la corresponsione delle indennità (indennità integrativa speciale prevista per legge ai lavoratori del comparto pubblico; indennità mensile di impiego operativo di base; indennità di missione; indennità di imbarco). Il terzo 
comma stabilisce che il cappellano militare "non percepisce compensi per lavoro straordinario in ordine all'assolvimento delle funzioni ministeriali in qualunque orario espletate". L'art. 10 stabilisce il trattamento previdenziale, indicando la cessazione del servizio al compimento dei 65 anni d'età come disposto dal successivo art. 13 secondo comma lett. b).

L'art. 11 riguarda la disciplina militare e l'applicazione della giurisdizione. Un regolamento disciplinare compatibile con la funzione dei cappellani sarà definito tramite decreto del Ministro della difesa di concerto con l'Ordinario militare (primo comma), che sarà emanato entro un anno dall'entrata in vigore dell'Intesa (art. 14, primo comma).

I cappellani non sono soggetti al Codice né alla disciplina militare né alla giurisdizione penale militare, se non in caso di mobilitazione, totale o parziale, o di servizio all'estero (secondo comma). Inoltre, si precisa che l'autorità giudiziaria, nel caso l'azione penale venga esercitata nei confronti di un cappellano militare, ne deve informare l'Ordinario militare (terzo comma).

L'art. 12 prevede le sanzioni nell'eventualità di infrazioni delle regole disciplinari e dei doveri di servizio, stabilendo anche la procedura per l'accertamento della violazione, con un'inchiesta formale affidata dal Ministero della difesa all' ",organo competente dell'Ordinariato militare", il quale formula una proposta motivata al ministro (secondo comma) che dovrà decidere sulla sanzione da infliggere (terzo comma).

Per l'art. 13 la sospensione dell'impiego, con privazione del trattamento economico, è prevista e applicata "di diritto" alle "sanzioni disciplinari ecclesiastiche che sospendono il Cappellano militare, dall'esercizio totale o parziale del ministero sacerdotale" (primo comma).

Il secondo comma della stessa disposizione tra le cause di cessazione dell'impiego indica anche alla lett. e) il caso di "revoca della designazione da parte dell'autorità ecclesiastica, e comunque qualora il Cappellano militare sia dimesso dallo stato clericale in applicazione delle norme canoniche".

Sull'art. 14 abbiamo già avuto modo di soffermarci supra al par. 4 per quanto riguarda il secondo comma (l'abrogazione delle norme dell'ordinamento militare con l'Intesa incompatibili) e il terzo comma (l'entrata in vigore dell'Intesa nell'ordinamento dello Stato e in quello della Chiesa cattolica). Vi è soltanto da aggiungere, quindi, che a norma del primo comma si dovrà procedere all'emanazione del regolamento disciplinare entro un anno. 


\section{7 - Le norme di adeguamento dell'ordinamento interno: novità e rilievi critici}

Le novità e i rilievi critici dell'Intesa li mettiamo in luce presentando la disciplina introdotta nell'ordinamento interno tramite l'operazione di riassetto normativo di cui all'art. 3 della legge n. 70 del $2021^{62}$.

\section{1 - La direzione e il coordinamento del Servizio di assistenza spirituale: una titolarità giuridica diretta in capo all'Ordinario militare per 1'Italia}

Sull'art. 17 C.O.M. ci siamo già ampiamente soffermati supra. Si pone, a riguardo, in evidenza solo che in questa disposizione, sia nella rubrica che nel testo della disposizione, non compare più il riferimento al "Servizio di assistenza spirituale" in quanto si fa riferimento direttamente all' "Assistenza spirituale". Mentre permane il riferimento al "Servizio di assistenza spirituale" nel novellato art. 1533 primo comma C.O.M. in cui non si parla più di "alta direzione" ma di "direzione e coordinamento".

In questa disposizione viene introdotta una modifica di non poco conto. Se $l^{\prime}$ "alta direzione" era "devoluta all'Ordinario militare per l'Italia63", la "direzione e il coordinamento", invece, "spettano all'Ordinario militare per $1^{\prime}$ Italia ${ }^{64 "}$. È chiaro che non si tratta di una differente novità linguistica. La scelta sottende una visione ben precisa dell'istituto dell'assistenza spirituale per i cattolici alle Forze armate nell'ordinamento repubblicano. Fino all'entrata in vigore dell'Intesa, la competenza in materia di direzione ("alta", come veniva definita) spettava principaliter allo Stato che, a norma di legge, la devolveva, cioè la trasferiva, all'Ordinario militare. In altre parole, lo Stato manteneva, almeno in astratto, una titolarità giuridica il cui esercizio veniva trasferito all'Ordinario militare. Ora, con la nuova disciplina, al contrario, nel rispetto delle obbligazioni assunte a livello internazionale, la legge riconosce la "spettanza" della diretta titolarità giuridica in materia di direzione e di coordinamento direttamente in capo all'Ordinario.

62 Nelle successive note a piè di pagina saranno proposte le corrispondenze tra le novelle apportate al C.O.M. e gli articoli dell'Intesa.

${ }^{63}$ Mio il corsivo.

${ }^{64}$ Mio il corsivo. 


\section{2 - L'introduzione della figura dei Cappellani militari coordinatori, in luogo degli Ispettori: alcuni problemi di sicurezza per le istituzioni militari dello Stato}

Nel secondo alinea della disposizione è stabilito che " 1 'Ordinario militare può avvalersi di cinque cappellani militari coordinatori presso gli Stati maggiori di Forza armata e i comandi generali" 65 . Pure in questo caso, si deve segnalare un ampliamento delle facoltà non più concesse ma spettanti all'Ordinario militare nella strutturazione della direzione dell'assistenza spirituale per i cattolici, che coinvolge anche le funzioni del Vicario generale. Nella disciplina previgente l'Ordinario militare era di diritto coadiuvato dal Vicario e da tre Ispettori, mentre ora è solo il Vicario generale che coadiuva l'Ordinario militare, il quale può decidere di dotarsi o meno dei cinque (o, eventualmente, anche meno) Cappellani militari coordinatori, la cui designazione è disciplinata dall'art. 1534-bis C.O.M.

$\mathrm{La}$ Curia rimane, quindi, istituzione soltanto canonica nell'organizzazione dell'Ordinario militare. Si deve precisare che la soppressione della figura dell'Ispettore dall'ordinamento civile ${ }^{66}$ non rende necessario, sul piano formale, l'adeguamento della legislazione canonica, in quanto gli Statuti dell'Ordinariato ${ }^{67}$ comunque prevedono all'art. 22 nella composizione della Curia i tre Ispettori insieme ad alcuni Vicari episcopali, al Cancelliere-Segretario generale addetto e ad altri Officiali addetti. Mentre il Cappellano militare coordinatore a oggi non ha una corrispondente istituzione canonica. A ragione di ciò, l'Ordinario potrebbe individuare in linea teorica i coordinatori in cappellani che, a livello canonico, non sono titolari di uffici nella Curia castrense pur acquisendo, nell'istituzione militare civile, funzioni determinanti al punto da accedere - a norma dell'art. 1534-bis C.O.M. - "liberamente ai luoghi militari di loro pertinenza"68. È indubbio che, senza un'adeguata riforma del diritto canonico particolare della Chiesa castrense in Italia, si potrebbero creare dislivelli normativi e istituzionali tra ordinamento canonico e ordinamento civile non indifferenti, in relazione ai poteri e alla posizione dell'Ordinario militare. È interessante, poi, sottolineare che nel successivo novellato secondo comma della disposizione in esame, mentre

\footnotetext{
${ }^{65}$ Cfr. art. 4, secondo comma, dell'Intesa.

66 La soppressione degli ispettori ha comportato la modifica di coordinamento degli articoli 1534, 1536, 1538, 1539, 1540, 1541, 1542, 1543, 1544, 1545 C.O.M.

67 Gli Statuti sono stati approvati dalla Santa Sede il 6 agosto 1987.

${ }^{68}$ Cfr. art. 2, quarto comma, dell'Intesa.
} 
viene modificata l'assimilazione di grado dell'Ordinario militare da "generale di corpo d'armata" a "tenente generale"69, mantenuto invariato il grado del Vicario in quello di "maggiore generale"70 ed eliminato ogni riferimento agli Ispettori, nulla viene specificato in relazione all'assimilazione di grado, la cui funzione - a differenza delle altre previste - non è assunta nell'inquadramento gerarchico del personale religioso ${ }^{71}$, al punto che secondo l'art. 1534-bis terzo comma C.O.M., "il conferimento dell'incarico di funzione ai Cappellani coordinatori non comporta alcuna modifica del trattamento economico".

Il Cappellano militare coordinatore non trova una corrispondenza sovrapponibile con la figura dell'Ispettore, per quanto riguarda la composizione della Commissione per la promozione dei cappellani militari ai sensi dell'art. 1609 C.O.M. Nella previgente disciplina la Commissione di avanzamento, presieduta dall'Ordinario, era composta dal Vicario generale militare, dai tre Ispettori e da un cappellano militare capo, con funzioni di segretario. Nella nuova composizione, così come è stato novellato l'art. 1609 C.O.M., invece, i tre ispettori sono sostituiti non dai Cappellani militari coordinatori bensì da tre secondi cappellani militari, di cui uno esercita le funzioni di segretario. A norma dell'art. 1609, secondo comma, C.O.M. "i secondi cappellani militari membri della commissione di avanzamento sono nominati dal Ministro della difesa, su proposta dell'Ordinario militare".

Insomma: nel C.O.M. ritroviamo una figura, quella del Cappellano militare coordinatore, in un numero massimo di cinque:

a) la cui eventuale individuazione pare di competenza strettamente fiduciaria dell'Ordinario militare, pure a livello canonico;

b) sulla cui procedura di nomina non viene detto nulla, così da ritenere quest'ultima attribuita in via esclusiva all'Ordinario militare, il quale deve solo limitarsi a comunicare il relativo nominativo al Ministro della difesa, a norma dell'art. 1534-bis, primo comma, C.O.M.72;

c) la cui posizione non è gerarchicamente inquadrata nell'ordinamento militare;

${ }^{69} \mathrm{Cfr}$. art. 6, primo comma, lett. a), dell'Intesa. Sul punto si veda infra par. 7.7.

${ }^{70}$ Cfr. art. 6, primo comma, lett. b), dell'Intesa.

${ }^{71}$ L'art. 6 dell'Intesa non fa riferimento al ruolo di Cappellano coordinatore presso gli Stati di Forza armata e i Comandi generali.

${ }^{72} \mathrm{Cfr}$. art. 4, secondo comma, dell'Intesa. 
d) che assolve a "compiti" (tanto l'Intesa quanto la legge, sul punto, sono molto generiche) affidatigli dall'Ordinario militare a norma dell'art. 1534-bis, secondo comma, C.O.M.73.

Eppure, nonostante ciò, il Cappellano militare coordinatore, come già è stato detto, a norma delle obbligazioni assunte dallo Stato italiano in sede internazionale, alle quali si è adeguata la normativa interna, può accedere "liberamente ai luoghi militari" 74 di sua pertinenza, vale a dire, in relazione agli Stati di Forza armata e ai Comandi generali cui è assegnato dall'Ordinario militare per adempiere a compiti non meglio precisati. Come non intravedere in questa situazione un problema, almeno in linea teorica, di sicurezza e di ordine pubblico per lo Stato? E, di converso, uno sconfinamento dell'Ordinario dall'ordo ecclesiae a quello della res publicae?

\section{3 - Il concetto canonico di "sede vacante" entra nell'ordinamento militare}

L'art. 1533, quarto comma, C.O.M. sulla giurisdizione ecclesiastica dell'Ordinario militare rimane invariato nella formulazione in quanto tale giurisdizione non subisce variazioni nell'Intesa ${ }^{75}$.

Il terzo comma dell'art. 1533 C.O.M. indica la "sede vacante" tra i casi in cui il Vicario generale sostituisce l'Ordinario: "Il Vicario generale militare sostituisce l'Ordinario militare nei casi di sede vacante, di assenza o di impedimento e lo rappresenta quando non può personalmente intervenire".

In tal modo, la disciplina statale si allinea perfettamente a quanto dispongono gli Statuti dell'Ordinariato all'art. 14: "In caso di sede vacante o impedita, l'Ordinariato Militare è retto dal Vicario Generale, al quale competono gli stessi diritti e doveri dell'Amministratore diocesano (cann. 409-430)". Essendo la "sede vacante" una nozione canonica, per la sua interpretazione in sede civile è necessario rimandare al can. 416 del Codice di diritto canonico (d'ora in avanti, c.i.c.), che diventa così operativo, in maniera implicita, nel nostro ordinamento: "La sede episcopale diviene vacante con la morte del Vescovo diocesano, con la rinuncia accettata dal Romano Pontefice, con il trasferimento e con la privazione intimata al Vescovo".

\footnotetext{
73 Ibidem.

${ }^{74}$ Mio il corsivo.

${ }^{75}$ Cfr. art. 1, secondo comma, dell'Intesa.
} 
Anche la causa dell',,impedimento" rimanda, pur non espressamente, alla nozione di sede impedita di cui al can. 412 c.i.c.:

"La sede episcopale si intende impedita se il Vescovo diocesano è totalmente impedito di esercitare l'ufficio pastorale nella diocesi a motivo di prigionia, confino, esilio o inabilità, non essendo in grado di comunicare nemmeno per lettera con i suoi diocesani".

\section{4 - La procedura di individuazione e di determinazione delle sedi per $i$ cappellani militari}

All'art. 1533 C.O.M. viene modificato totalmente il quinto comma e introdotto un sesto comma. Questi due commi riguardano le procedure, rispettivamente, di determinazione delle sedi ${ }^{76}$, da parte del Ministro della difesa, e di individuazione della sede per ciascun cappellano militare ${ }^{77}$, da parte dell'Ordinario. Si segnala che, per quanto attiene alla determinazione delle sedi, il quinto comma prescrive che il Ministro della difesa deve agire "di concerto" con il Ministro dell'economia e delle finanze nel caso in cui si tratta del Corpo della Guardia di Finanza. Tale passaggio non è stato concordato in sede bilaterale con la Santa Sede in quanto non compare nell'Intesa. Si tratta insomma di un'interposizione legislativa di natura unilaterale all'interno di una norma che adegua l'ordinamento domestico a impegni internazionali. Da un punto di vista di tecnica della normazione, ciò non sembra molto corretto.

\section{5 - L'art. 1533-bis C.O.M.: il divieto di corresponsione di emolumenti accessori e la materia "spirituale e pastorale"}

È poi introdotto l'art. 1533-bis C.O.M. che riguarda lo svolgimento del "Servizio di assistenza spirituale" (anche qui il ritorna il riferimento al "servizio") consistente, per i cappellani militari, come specificato al primo comma, nel curare "la celebrazione dei riti liturgici, la catechesi, nonché l'organizzazione di ogni opportuna attività pastorale, anche oltre l'orario servizio, senza oneri aggiuntivi per l'amministrazione"78.

È importante quest'ultima specificazione, in quanto per i cappellani militari non è più prevista la corresponsione di emolumenti accessori, come il compenso per il lavoro straordinario, il compenso forfettario di

\footnotetext{
${ }^{76}$ Cfr. art. 4, terzo comma, dell'Intesa.

77 Ibidem.

${ }^{78}$ Cfr. art. 1, terzo comma, dell'Intesa.
} 
impiego e gli emolumenti ex art. 1826-bis C.O.M. (ciò ovviamente non esclude gli eventuali obblighi assicurativi in ordine all'assolvimento delle funzioni ministeriali espletate in qualunque orario) ${ }^{79}$. Rimangono ferme, comunque, l'indennità integrativa speciale, l'indennità mensile di impiego operativo di base (ma senza alcuna maggiorazione) e, eventualmente, l'indennità di missione e l'indennità di imbarco.

Il secondo comma della norma in esame dispone che per la materia "propriamente spirituale e pastorale" i cappellani militari devono osservare le norme dell'ordinamento canonico nonché le "direttive dell'Ordinario militare" 80 . La materia "spirituale e pastorale" riguarda le funzioni di parroco che svolgono i cappellani ${ }^{81}$. Il terzo comma si riferisce alla possibilità, per i cappellani militari, di avvalersi ai "fini delle attività di culto" della collaborazione di sacerdoti in servizio presso la diocesi competente per territorio, previa comunicazione alle autorità militari da parte dell'Ordinario militare, senza oneri aggiuntivi a carico dell'amministrazione ${ }^{82}$. Per definire le attività di culto si potrebbe ricorrere alla nozione di cui all'art. 16 lett. a) della legge n. 222 del 1985 in merito al riconoscimento degli enti ecclesiastici: "attività di religione o di culto quelle dirette all'esercizio del culto e alla cura delle anime, alla formazione del clero e dei religiosi, a scopi missionari, alla catechesi, all'educazione cristiana".

Al quarto comma si dispone che "in caso di assenza, il cappellano militare è sostituito dal parroco competente per la sede di servizio, previa comunicazione dell'Ordinario militare al comandante della sede, senza oneri aggiuntivi per l'Amministrazione" ${ }^{\prime \prime 3}$. Ma quest'ultima disposizione appare vaga. Nulla è aggiunto riguardo a che cosa debba intendersi per "assenza", anche sotto il profilo di determinazione dei termini temporali, e quali facoltà comporti questa sostituzione nella sede di servizio. È sufficiente una comunicazione da parte dell'Ordinario militare all'Amministrazione per dare la possibilità a un soggetto esterno

79 Cfr. art. 9, terzo comma, dell'Intesa. La Relazione tecnica precisa che la mancata corresponsione ai cappellani militari di tali emolumenti non determina in sé un risparmio per il bilancio dello Stato, in quanto gli stanziamenti non vengono ridotti in funzione della mancata erogazione a favore dei cappellani. Vedi A.C. 2657. Verifica delle quantificazioni, n. 301, 26 gennaio 2021, p. 15 (in https://documenti.camera.it/Leg18/Dossier/ Pdf/VQ2657.Pdf - consultato l'ultima volta il 12 settembre 2021).

${ }^{80} \mathrm{Cfr}$. art. 1, quinto comma, dell'Intesa.

${ }^{81} \mathrm{Cfr}$. art. 1, quarto comma, dell'Intesa.

82 Cfr. art. 3, primo comma, dell'Intesa.

${ }^{83} \mathrm{Cfr}$. art. 3, secondo comma, dell'Intesa. 
all'organizzazione militare - ancorché sacerdote - di muoversi, per ragioni di servizio spirituale, all'interno dei luoghi militari. L'automatismo relativo al parroco competente della sede servizio comporta, inoltre, diversi problemi sul piano canonistico. Sacerdoti non idonei a svolgere l'incarico di cappellano militare potrebbero ritrovarsi direttamente coinvolti nel servizio di assistenza spirituale alle Forze armate. Inoltre, il parroco eserciterebbe la cura pastorale su una comunità che, sotto il profilo soggettivo, è estranea all'autorità del vescovo diocesano, differentemente da quanto prescrive il can. 519 c.i.c.:

"Il parroco è il pastore proprio della parrocchia affidatagli, esercitando la cura pastorale di quella comunità sotto l'autorità del Vescovo diocesano, con il quale è chiamato a partecipare al ministero di Cristo, per compiere al servizio della comunità le funzioni di insegnare, santificare e governare, anche con la collaborazione di altri presbiteri o diaconi e con l'apporto dei fedeli laici, a norma del diritto".

In tal modo, la potestà dell'Ordinario militare si estende, in maniera automatica, finanche a sacerdoti che non sono incardinati nell'Ordinariato, andando a interferire con quella del vescovo diocesano.

Il quinto comma della disposizione, infine, dà un'ulteriore facoltà all'Ordinario militare: quella di dispensare dall'obbligo dei cappellani militari di risiedere in una delle sedi di servizio, il cui alloggio deve essere garantito dall'amministrazione di appartenenza ${ }^{84}$.

\section{6 - La nomina dell'Ordinario militare e del Vicario generale}

Il nuovo art. 1534 C.O.M. riguarda la nomina dell'Ordinario militare e del Vicario Generale, entrambe di prerogativa del Presidente della Repubblica ma su designazione, rispettivamente, della Santa Sede ${ }^{85}$ e dell'Ordinario militare ${ }^{86}$. In questo caso, non viene introdotta una modifica sostanziale alla disciplina previgente. Ma vi è da sottolineare che nel testo dell'Intesa non compare la previsione che, invece troviamo nella norma, della "proposta del presidente del Consiglio dei ministri, di concerto con il Ministro dell'interno e con il Ministro della difesa". Il passaggio, non concordato in sede bilaterale (e, quindi, la Chiesa cattolica potrebbe considerarlo anche un'indebita ingerenza), risponde più alle esigenze di

\footnotetext{
${ }^{84}$ Cfr. art. 2, secondo comma, dell'Intesa.

85 Cfr. art. 4, primo comma, dell'Intesa.

86 Ibidem.
} 
una logica ormai anacronistica, di stampo evidentemente giurisdizionalistico, con necessità di rigore formalistico ma non sostanziali.

\section{7 - Il regime di assimilazione ai gradi gerarchici per i cappellani militare: nella sostanza non cambia nulla}

Potrebbe sembrare che una prima innovazione riguardo ai gradi sia la previsione contenuta all'art. 1555 secondo comma, C.O.M. in cui l'Ordinariato militare è assimilato al grado non più di Generale di corpo d'armata ma di tenente generale. In realtà, questa non è un'innovazione. Perché quello di Generale di corpo d'armata è un livello di comando che corrisponde esattamente al grado di tenente generale ${ }^{87}$. Era la previgente normativa che semplicemente generava confusione tra livello di comando e grado gerarchico. Insomma, non cambia nulla per lo status giuridico dell'Ordinario militare.

Poi, il nuovo art. 1546, primo comma, C.O.M. ridefinisce i gradi gerarchici dei cappellani militari ai sensi dell' art. 6 dell'Intesa: Ordinario militare assimilato al rango di tenente generale, Vicario generale militare come maggiore generale, Secondo cappellano militare come tenente colonnello (per non oltre dieci unità), Primo cappellano militare capo come Maggiore spe, Cappellano militare capo come capitano spe, Capo militare addetto come tenente spe, Cappellano militare di complemento come sottotenente di complemento.

Il secondo comma della disposizione, poi, specifica che $\mathrm{l}^{\prime}$,"attribuzione dei gradi gerarchici" avviene per "assimilazione di rango ai gradi militari".

La questione sull' ",assimilazione" dei gradi gerarchici è presentata come una delle principali novità o, meglio, come uno degli "elementi qualificanti" dell'Intesa. D'altronde si tratta di uno degli aspetti sui quali maggiormente si concentra l'attenzione dell'opinione pubblica, in quanto icasticamente rappresenta l'integrazione organica di sacerdoti cattolici nella struttura militare.

Nella Relazione tecnica si può leggere:

"Tra gli elementi qualificanti dell'Intesa si segnala la ridefinizione, per i cappellani militari, del concetto di assimilazione ai gradi gerarchici rispetto alla precedente disciplina e il relativo rapporto con le strutture e le funzioni militari. Resta fermo il rapporto organico che

87 C.M. DECHIGI, I gradi dell'esercito italiano. Distintivi di incarico e funzionali, Esercito italiano, 2011, p. 18. 
lega i cappellani delle strutture militari, per consentire la piena agibilità di spazi e luoghi nell'ambito dell'organizzazione militare, ma il cappellano militare non si configura più come un militare tra $\mathrm{i}$ militari, soggetto in tutto e per tutto alle norme e alla disciplina militare, acquistano, invece, centralità le funzioni spirituali e pastorali che contraddistinguono tale figura peculiare e autonoma" 88 .

\section{Così, a riguardo, si esprimeva Dalla Torre:}

"[...] il testo dell'intesa supera la immedesimazione strutturale e giuridica del cappellano nell'apparato militare, che nel novecento costituiva un paradigma tipico nelle normative degli stati in materia, pur assicurandogli un rapporto organico con quella realtà. in sostanza, il cappellano militare viene ad avere uno stato giuridico che, quanto ai gradi militari, ne comporta solo l'assimilazione. Tale nuova configurazione dello stato giuridico, basata sull'assimilazione e non sull'integrazione nella gerarchia militare, comporta una serie di conseguenze, tra cui: nell'apparato militare non ha poteri di comando o di direzione, né di amministrazione; non porta armi né, di regola, indossa la divisa militare, essendo tenuto a vestire l'abito ecclesiastico proprio; di norma è sottratto al codice di disciplina militare e sottoposto ad un Regolamento disciplinare proprio, prevedente obblighi peculiari e sanzioni specifiche, compatibili con la natura delle funzioni da lui svolte. in sintesi, questo regime consente al cappellano di muoversi liberamente all'interno di una struttura fortemente gerarchizzata, rendendo un servizio pienamente fruibile" 89 .

Si riprende, in questa direzione, la nuova formulazione dell'art. 1555 C.O.M., con la modifica del secondo comma e l'inserimento dei commi terzo e quarto:

“2. I cappellani militari sono soggetti alle specifiche disposizioni disciplinari contenute in un regolamento definito con decreto del Ministro della difesa di concerto con l'Ordinario militare fatto salvo quanto previsto dalla sezione IX.

3. L'autorità giudiziaria, in caso di esercizio dell'azione penale nei confronti di un cappellano militare, ne informa l'Ordinario militare.

4. I cappellani militari non portano armi e indossano, di regola, l'abito ecclesiastico loro proprio, salvo situazioni speciali elle quali sia necessario indossare la divisa militare ${ }^{\prime \prime 90}$.

88 Cfr. la Relazione tecnica tra i documenti del Dossier presentato al Senato della Repubblica, Atto parlamentare n. 1763, p. 14.

89 G. DALLA TORRE, Nuova intesa, cit., p. 414.

90 In questa norma confluiscono diverse disposizioni dell'Intesa: il secondo comma 
In realtà, la distanza tra "nuovo" e "vecchio" regime sul piano sostanziale non è così evidente come viene presentata. Difatti, come precisato nella relazione tecnica, il concetto di "assimilazione" non preclude il "rapporto organico che lega i cappellani alle strutture militari". E, quindi, a ben vedere non viene superata la "immedesimazione strutturale e giuridica del cappellano nell'apparato militare", come, invece, sostenuto da Dalla Torre. Quello che viene considerato quale elemento "qualificante" dell'Intesa altro non è che la riproposizione dello stesso modello di integrazione. Infatti, già nella previgente disciplina si aveva un'assimilazione di rango, come dimostra la l'originaria formulazione dell'art. 1546 C.O.M.:

"terzo cappellano militare capo, assimilato di rango al grado di tenente colonnello; secondo cappellano militare capo, assimilato di rango al grado di tenente colonnello; primo cappellano militare capo, assimilato di rango al grado di maggiore $[\ldots]^{\prime \prime 91}$.

L'ipotesi secondo cui l'assimilazione di grado non è una novità rispetto al precedente regime è confermata anche da un preciso contesto normativo:

a) la perdurante vigenza dell'art. 1551, terzo comma, C.O.M.: "i cappellani militari in congedo assoluto non hanno più obblighi di servizio, ma conservano il grado e l'onore dell'uniforme e sono soggetti alle disposizioni del presente codice riflettenti il grado e la disciplina".

b) ai sensi dell'articolo 1552 C.O.M. rimane l'inquadramento nei "tre ruoli unici per tutte le Forze armate dello Stato" (primo comma), effettuato "in ordine decrescente di grado e di anzianità" (secondo comma) 92.

c) la perdurante vigenza dell'art. 1553 C.O.M. circa l'anzianità di grado che, ai sensi dell'art. 854 C.O.M., "determina la precedenza di un militare rispetto ai pari grado";

d) l'attuale art. 1597 C.O.M. sulle "cause di perdita del grado";

e) l'art. 1599, primo comma, lett. b), C.O.M. che individua tra le sanzioni disciplinari da applicare eventualmente al cappellano militare anche la "sospensione dalle funzioni del grado" rimanendo fermo l'art.

richiama l'art. 11, primo comma; il terzo comma l'art 11, terzo comma; il quarto comma l'art. 5, terzo comma. Come già precedentemente sottolineato, a norma dell'art. 14, primo comma, dell'Intesa il regolamento di cui al secondo comma dell'art. 1555 C.O.M. sarà predisposto entro un anno dall'entrata in vigore delle disposizioni.

91 Miei i tre corsivi.

92 Per la definizione di "ruolo" si rimanda all'art. 790 C.O.M. 
1589 C.O.M. sulla sospensione dalle funzioni del grado per motivi precauzionali, disciplinari, penali;

f) la previsione che l'organo inquirente dell'Ordinario militare, ai fini dell'inchiesta formale per l'accertamento di un'infrazione disciplinare, "deve essere di grado o anzianità superiore all'inquisito" 1602, terzo comma, C.O.M.;

g) le forme di avanzamento, per anzianità congiunta al merito e per merito comparativo, il riferimento al grado, ai sensi dell'art. 1611 C.O.M.

Né può ritenersi che la causa di "perdita del grado" sia stata ormai espunta dall'art. 1577 C.O.M. In questa disposizione sono ora analiticamente ricomprese le cause di perdita del grado ai sensi dell'art. 1597 C.O.M. Ne consegue che, nonostante il meccanismo quasi a "matrioska"94, il risultato non cambia.

Insomma, sotto un profilo strettamente normativo, l'assimilazione ai gradi non è una novità per i cappellani militari. I cappellani sono sempre stati assimilati all'organizzazione militare. Anzi, il loro rapporto organico con l'amministrazione militare è esattamente determinato dall'assimilazione. Non vi è un carattere meramente onorifico, cioè una semplice assimilazione di rango, ma ci troviamo di fronte a una vera e propria assimilazione di status, con l'attribuzione di diritti e di doveri propri dei militari.

Il concetto di appartenente alle Forze armate è di per sé molto ampio ed è prevalentemente ricostruito facendo ricorso alla legislazione penale militare: a norma dell'art. 10 del Codice Penale Militare di Pace (regio decreto n. 303 del 1941; C.P.M.P.) "la legge penale militare si applica agli assimilati ai militari [...]", e la dottrina giuridica ha sempre pacificamente individuato negli "assimilati" i cappellani militari ${ }^{95}$, nei confronti dei quali la legge penale militare si applica nei casi di mobilitazione e di missioni all'estero, ai sensi dell'art. 1555 C.O.M.

Una più dettagliata specificazione delle funzioni e delle attività di carattere pastorale e spirituale dei cappellani militari non incide, nella forma come nella sostanza, su una disciplina normativa che mantiene inalterata la forte integrazione dei sacerdoti cattolici nelle Forze armate;

93 Mio il corsivo.

94 Seppure con riferimento ad altro tema, che esula dall'oggetto del presente saggio, ritroviamo l'immagine della "matrioska normativa" in A. TIRA, Normativa emergenziale ed esercizio pubblico del culto. Dai protocolli con le confessioni diverse dalla cattolica alla legge 22 maggio 2020, n. 35, in Giustizia insieme, 8 giugno 2020.

95 Vedi, per tutti, F. BASSETTA, Lineamenti di diritto militare, 2a ed., Laurus, Roma, 2012, p. 209. 
un'integrazione di "tipo economico, gerarchico e disciplinare" non coerente "né con lo Stato laico né con la funzione spirituale affidata ai cappellani"96.

\section{8 - Altre novelle legislative: l'organico dei cappellani; la procedura di nomina; la dismissione dallo stato clericale}

L'art. 1547 C.O.M. è novellato con l'inserimento del secondo comma che fissa l'organico dei cappellani militari a 162 unità, per "garantire un rapporto di proporzionalità" come leggiamo nell'art. 5, primo comma, dell'Intesa.

Le modifiche all'art. 1549 C.O.M. si riferiscono, poi, alla procedura di nomina dei cappellani di complemento, riprendendo quanto già disposto all'art. 17 C.O.M. Se nella previgente disciplina il cappellano veniva nominato dal Presidente della Repubblica, su proposta del Ministro della difesa e su designazione dell'Ordinario militare, ora il cappellano è direttamente nominato con un decreto del Ministro della difesa, previa designazione dell'Ordinario militare ${ }^{97}$.

L'art. 1559 C.O.M. riguarda la nomina da cappellano militare addetto in servizio permanente alle funzioni cappellano di complemento: dopo avere presentato apposita domanda (primo comma, lett. a), si deve aver prestato servizio continuativo almeno per cinque anni, riportando la qualifica di ottimo (primo comma, lett. b) e non aver superato il $45^{\text {esimo }}$ anno di età (primo comma, lett. c).

La novella contenuta al secondo comma dell'art. 1560 C.O.M. pone ancora problemi di adempimento delle obbligazioni internazionali in quanto non riproduce fedelmente ciò che prescrive l'Intesa. Infatti, a norma dell'art. 8, terzo comma, dell'Intesa il rapporto "può essere interrotto, sospeso o cessare solo in base alle disposizione della presente Intesa"; mentre a norma dell'art. 1560, secondo comma, C.O.M. l'impiego "non può essere interrotto, sospeso o cessare se non nei casi e nei modi stabiliti dal presente Codice". Insomma: se l'interruzione, la sospensione o la cessazione del servizio, ai sensi dell'Intesa, devono essere rapportati in base alle disposizioni dell'Intesa, nella norma statale i casi e modi sono stabiliti dal Codice. Inoltre, l'art. 1561, primo comma, C.O.M. prescrive che per la sospensione "precauzionale" dell'impiego devono osservarsi, "in

96 C. CARDIA, Manuale di diritto ecclesiastico, il Mulino, Bologna, 1999, p. 424.

${ }_{97}$ Cfr. art. 1, primo comma, dell'Intesa. 
quanto applicabili, le disposizioni "della sezione IV del capo III del titolo V del libro IV".

All'art. 1577, primo comma, C.O.M. sono inserite tre nuove cause di cessazione del servizio permanente: i "motivi disciplinari", al pari di quanto ribadisce anche la novella dell'art. 1599 C.O.M.; la "revoca della designazione da parte dell'autorità ecclesiastica"; le "dimissioni dallo stato clericale, in applicazione delle norme canoniche"

Come già detto, queste cause sono corrispondenti in realtà alle cause analitiche di perdita del grado ai sensi dell'art. 1597 C.O.M. Quella che nella previgente disciplina era identificata come la "inidoneità permanente alle funzioni sacerdotali", in senso atecnico, ora viene compresa, con maggiore precisione, nelle "dimissioni dallo stato clericale", trovando operatività nell'ordinamento italiano il can. 290 c.i.c. ${ }^{99}$.

Inoltre, la cessazione del cappellano per dismissione dallo stato clericale, per perdita dei diritti civili e politici o per cessazione dal servizio per motivi disciplinari comporta la perdita del grado, ai sensi dell'art. 1597 C.O.M.

L'art. 1601 C.O.M. concerne l'avvio dell'inchiesta formale ai fini dell'accertamento di un'infrazione disciplinare su rapporto dell'autorità competente ${ }^{100}$. La previsione di un cappellano militare inquirente prevista dalla previgente disciplina è ora sostituita, nel nuovo art. 1602 C.O.M., dalla previsione di un "organo competente dell'Ordinariato militare"101, anche se, poi, al terzo comma, ritorna il principio secondo cui "l'inquirente deve essere di grado o anzianità superiore all'inquisito", ma è eliminata la possibilità residuale, nel caso in cui non fosse possibile rintracciare un inquirente di grado o anzianità superiore all'inquisito, di affidare l'inchiesta formale a un ufficiale dell'esercito di grado superiore all'inquisito. L'inquirente ora, ai sensi del quarto comma, deve anche formulare proposta motivata nel momento in cui rimette il rapporto conclusivo al Ministro ${ }^{102}$. L'art. 1603 C.O.M. stabilisce che il Ministro "in base alle risultanze dell'inchiesta formale, decide, sentito il parere dell'Ordinario militare, se al cappellano militare deve essere o meno inflitta una delle sanzioni disciplinari [...]".

98 Cfr. art. 13, secondo comma, dell'Intesa.

99 Cfr., sul can. 290 c.i.c., S. BERLINGÒ, M. TIGANO, Lezioni di diritto canonico, Giappichelli, Torino, 2008, pp. 166-168.

100 Cfr. art. 12, secondo comma, dell'Intesa.

101 Cfr. art. 12, terzo comma, dell'Intesa.

102 Ibidem. 


\section{8 - Prime tensioni tra Ordinariato militare e amministrazione della Difesa sull'applicazione della legge}

La legge n. 70 in commento è entrata in vigore il 23 maggio 2021, vale a dire "il giorno successivo a quella sua pubblicazione nella Gazzetta Ufficiale", secondo quanto prescritto dal legislatore all'art. 7.

In data 27 maggio il direttore del Centro Unico Stipendiale Interforze dello Stato Maggiore della Difesa, maggiore generale Salvatore Vergari, ha inviato una comunicazione ai Centri Stipendiali territoriali per sottolineare che la legge interviene anche sul trattamento economico dell'Ordinario militare e dei cappellani militari, evidenziando la necessità di un "immediato adeguamento dei trattamenti economici attribuiti al personale"103.

Conseguentemente a questa comunicazione, il 4 giugno 2021 il Capo del I Reparto Personale dello Stato Maggiore della Difesa, ammiraglio di divisione Giacinto Ottaviani, ha diramato due circolari contenenti disposizioni applicative. Con la prima si è risposto a una richiesta di chiarimenti da parte dell'Ordinariato militare in materia di attribuzione del trattamento economico accessorio relativo al compenso per lavoro straordinario ai sensi dell'art. 1533-bis, primo comma, C.O.M. La norma, viene sottolineato nella circolare,

"riconduce le funzioni ministeriali dei cappellani militari ai servizi precipui della 'competenza parrocchiale' e, tal fine, rientrano i riti liturgici (le celebrazioni dei Sacramenti e dei Sacramentali), la catechesi e l'organizzazione di ogni opportuna attività pastorale. Ciò posto, l'esercizio delle suddette funzioni oltre il normale orario di servizio, previsto per l'Ente di appartenenza, non comporterà la corresponsione di emolumenti per lavoro straordinario"104.

Con la seconda si è data risposta a un'ulteriore richiesta di chiarimenti da parte dell'Ordinario militare per l'Italia in merito al servizio svolto dai "sacerdoti collaboratori", ai sensi dell'art. 1533-bis, terzo comma, C.O.M., precisando che tale personale:

"- assume canonicamente la denominazione di cappellano (art. 2, can.

564 e seguenti del Codice di Diritto Canonico);

103 Il documento è stato pubblicato sul sito internet del Partito per la tutela dei Diritti dei militari e delle forze di polizia (PDM) al seguente link: https://www. partitodirittimilitari.it/wpcontent/uploads/2021/06/Allegati_1_2_e_3.pdf(Allegato 1).

104 Il documento è stato pubblicato sul sito internet del Partito per la tutela dei Diritti dei militari e delle forze di polizia (PDM) al seguente link: https://www. partitodirittimilitari.it/wpcontent/uploads/2021/06/Allegati_1_2_e_3.pdf(Allegato 2). 
- è incardinato nell'Ordinariato Militare per l'Italia, in una Diocesi o in un Istituto Religioso;

- riceve l'incarico canonico dell'Ordinario Militare per l'Italia, previa concessione del nulla osta da parte del proprio Ordinario ove il Diritto Canonico lo richieda"105.

Alla luce della comunicazione del Centro Stipendiale e delle disposizioni applicative diramante dal Reparto Personale, il Vicario generale militare ha chiesto ai Generali e agli Ammiragli di disporre che «tutti i Comandi degli Enti Militari che hanno in forza i Cappellani Militari attendano le "norme esplicative" della Legge in oggetto, che saranno redatte in accordo con $\mathrm{l}^{\prime}$ Ordinariato Militare per 1'Italia»106.

Le "norme esplicative" a cui si riferisce l'Ordinariato militare sembrano richiamare la necessità di decreti attuativi da parte della competente amministrazione dello Stato. La legge in questione, però, non prevede tale possibilità. Le norme d'altronde appaiono redatte in maniera sufficientemente chiara e precettiva, al punto da non aver bisogno di ulteriori provvedimenti per la loro applicazione concreta. Ancor di più, la legge, come abbiamo visto, prevedeva un termine di vacatio legis di solo un giorno. Trascorso tale termine, dal 23 maggio, la legge è entrata in vigore, è obbligatoria e deve essere osservata dai cittadini come dagli organi dello Stato, in maniera "immediata", come è stato sottolineato dalla direzione del Centro Unico Stipendiale Interforze dello Stato Maggiore della Difesa. $\mathrm{Ne}$ consegue che eventuali emolumenti accessori percepiti dai cappellani militari dopo l'entrata in vigore della legge sono da considerarsi illegittimi.

\section{9 - Perché nell'Intesa non c'è alcun riferimento alle donne consacrate che operano negli stabilimenti militari?}

Il titolo III del libro V del C.O.M. al Capo II e alla sezione II del Capo III fa espresso riferimento alle religiose in servizio presso gli stabilimenti sanitari militari (artt. 1620 e 1623). Queste disposizioni non sono state novellate dalla legge n. 70 del 2021, in quanto l'Intesa tra Santa Sede e

105 Il documento è stato pubblicato sul sito internet del Partito per la tutela dei Diritti dei militari e delle forze di polizia (PDM) al seguente link: https://www.partito dirittimilitari.it/wpcontent/uploads/2021/06/Allegati_1_2_e_3.pdf(Allegato 3).

106 Il documento è stato pubblicato sul sito internet del Partito per la tutela dei Diritti dei militari e delle forze di polizia (PDM) al seguente link: https://www.partito dirittimilitari.it/wpcontent/uploads/2021/06/lettera-Frigerio.pdf. 
Governo italiano non interviene sullo status giuridico delle religiose impegnate negli stabilimenti sanitari militari. Eppure, le stesse religiose, anche alla luce della collocazione sistematica del Codice, rientrano tra il personale religioso, pur non essendo sotto la competenza diretta dell'Ordinariato militare, in quanto a norma dell'art. 1620 C.O.M. l'assunzione delle suore è regolamentata da apposite convenzioni da stipularsi tra l'ospedale militare sanitario interessato e la Casa madre a cui appartengono le religiose. Il ruolo che le religiose svolgono negli stabilimenti sanitari militari è una componente fondamentale dell'assistenza spirituale. Ci si domanda, quindi, come mai un accordo bilaterale volto a disciplinare la materia dell'assistenza spirituale nelle Forze armate non si occupi delle religiose.

La questione, probabilmente, rappresenta solo la punta di un iceberg di un problema molto più ampio e diffuso nella Chiesa cattolica, che riguarda la questione di genere $\mathrm{e}$, in particolare, il ruolo delle consacrate ${ }^{107}$. Un ruolo che certamente andrebbe maggiormente tutelato e valorizzato nell'ordinamento canonico e, di riflesso, nella politica e nel diritto concordatari. L'esigenza di diritti, di regole e di contratti è improcrastinabile, come ha detto anche suor Maryanne Loughry, docente al Boston College, in un'intervista sul mensile "Donna, Chiesa, Mondo" de L'Osservatore Romano:

"Nella Chiesa ci sono molte cose date per assodate: che non siamo molto generosi, che usciamo dagli schemi se c'è da fare qualcosa di speciale. Non voglio rinunciare a questa caratteristica, ma penso che a volte venga sfruttata [...] nella Chiesa c'è la questione del clericalismo e il controllo sulle parrocchiane e sulle religiose sta diventando fonte di tensione in alcune aree [...] la nostra Chiesa è molto gerarchica, qualcuno di recente ha usato il termine gender blind: si fatica a comprendere il nostro contributo e come ci sentiamo quando non siamo riconosciute o ascoltate. Stiamo avendo più leader donne nella politica mondiale e la Chiesa non sopravvivrà se non si adatta"108.

Probabilmente, insomma, per ciò che riguarda la revisione dell'istituto dell'assistenza religiosa alle Forze armate, da parte vaticana, non si è pensato che una componente fondamentale dell'assistenza religiosa sia rappresentata dalle suore. Negli ambienti vaticani hanno

\footnotetext{
107 Sul tema, in una prospettiva di diritto, religione e letteratura, è interessante rinviare al romanzo di L. SCARAFFIA, La donna cardinale, Marsilio, Venezia, 2020.

${ }^{108}$ F. RE DAVID, Diritti, regole, contratti pure se si lavora per la Chiesa. Suor Maryanne Loughry: una questione che va affrontata, in L'Osservatore Romano - Donne, Chiesa, Mondo, ottobre 2021, pp. 18-19.
} 
pensato soltanto ai preti, ma non alle suore, e questo si è riverberato anche nelle scelte governative.

Ciò anche se nel 2014 un gruppo di suore avesse partecipato al Corso di formazione dell'Ordinariato militare e, all'epoca, l'Ordinario militare non avesse esclusa la possibilità di riconoscere alle donne consacrate uno spazio di autonomia nell'assistenza spirituale alle Forze armate:

\begin{abstract}
"[...] al fine di una migliore cura spirituale di donne e uomini militari, non si può che incoraggiare, accanto alla necessaria figura dei sacerdoti, la presenza di religiose e donne consacrate le quali, come in qualsiasi comunità cristiana, possono portare la ricchezza dei loro carismi, delle loro competenze, del valore stesso della femminilità e della maternità"109.
\end{abstract}

La revisione del servizio di assistenza religiosa per i cattolici alle Forze armate avrebbe potuto rappresentare l'occasione di dare a seguito a quanto auspicato dall'Ordinario militare e, quindi, di offrire un adeguato riconoscimento normativo alle religiose impegnate nell'assistenza spirituale alle Forze armate. Ma così non è stato. E quindi la presenza di queste religiose negli stabilimenti sanitari militari continua a essere disciplinata unilateralmente dallo Stato.

D'altro canto, la valorizzazione del ruolo delle suore andrebbe anche nella direzione di un adeguamento dell'istituto all'entrata in servizio di personale femminile con la legge n. 380 del 1999.

\title{
10 - Conclusioni: il Capo I della legge n. 70 del 2021 come "caso-studio" del decadimento normativo e della crisi del Parlamento
}

L'Intesa di cui alla legge n. 70 del 2021 è stata presentata come dettata dall'esigenza di fare risparmiare le casse dello Stato. Sembra però che il risparmio non sia così rilevante, andando dai 3,7 milioni di euro nel 2021 ai 2,2 milioni nel 2029110. Ma non è comunque questo il problema:

109 Cfr. G. GALEAZZI, Sei suore al corso per cappellano militare, in La Stampa, 28 luglio 2014 (https://www.lastampa.it/vatican-insider/it/2014/07/28/news/sei-suore-al-corso-per-cappella no-militare-1.35737870 - ultima consultazione: 5 novembre 2021).

110 F. PERROTTA, Libertà religiosa, cit., p. 4. Il risparmio, quindi, pare non sia sufficiente a realizzare quanto auspicato in A. DE OTO, Altare e mostrine, cit., p. 57: “[...] l'opportunità di destinare la somma risparmiata con questo accordo alla creazione di una cappellania strutturata ed incardinata nel Ministero della Difesa, diversa da quella di religione cattolica, indipendente a livello operativo e dialogante con l'Ordinariato 
rimaniamo convinti che non possono essere criteri economicistici a determinare scelte di politica del diritto che incidono sull'affermazione dei diritti fondamentali111.

Non può che destare preoccupazione il fatto che la legge $n .70$ del 2021, come si è visto, presenti incertezze e diverse problemi strutturali dal punto di vista della tecnica e della redazione normativa, anche in relazione agli obiettivi che intende conseguire. Questo provvedimento appare paradigmatico, al punto da poter essere considerato un vero e proprio "caso-studio", del processo di "inflazione legislativa"112 e di crisi della legge statale - sempre più "oscura"113 -, caratterizzato dallo "scadimento della qualità della stessa, mal redatta, spesso modificata, stratificata, scarsamente fattibile"114. Nello specifico, non può che registrarsi, inoltre, una poca accuratezza nel corso dei lavori parlamentari. Ad esempio, nel Dossier preparato dal Dipartimento Difesa il 23 ottobre 2020, come scheda di lettura per l'esame nell'assemblea della Camera dei deputati, si legge: "I cappellani sono inseriti a pieno titolo, come sacerdoti che prestano servizio in favore della diocesi appartenenza, nel sistema economico di sostentamento del clero"115.

Ma di una simile innovazione, che pure sarebbe stata auspicabile ${ }^{116}$, non c'è traccia nell'articolato normativo; d'altronde, l'Ordinariato militare, pur avendo un Ufficio economato, ancora difetta di una tipica istituzione diocesana, quale $1^{\prime}$ Ufficio per il sostentamento del clero ${ }^{117}$. Inoltre, nella

militare [...], chiudendo il cerchio e dando piena attuazione all'art. 19 della Costituzione e a quel pluralismo che diverrebbe così davvero pieno [...]".

111 Sul punto, si rimanda a L.M. GUZZO, L'assistenza religiosa, cit., pp. XXIV-XXVI.

112 Di "inflazione giuridica" parlava già F. CARNELUTTI, La morte del diritto, in AA. VV., La crisi del diritto, Cedam, Padova, 1977, p. 180: "più cresce il numero delle leggi giuridiche e più diminuisce la possibilità della loro accurata e ponderata formazione. L'analogia, sotto questo aspetto, tra l'inflazione legislativa e l'inflazione monetaria, da me usata più volte, è decisiva".

${ }^{113}$ Cfr. M. AINIS, La legge oscura. Come e perché non funziona, Laterza, Roma-Bari, 2002.

114 C. MEOLI, Il declino della legge statale, in Treccani.it, 2013 (https://www.treccani.it/en ciclopedia/il-declino-della-legge-statale_\%28Il-Libro-dell\%27anno-del-Diritto\%29/ - ultima consultazione: 6 novembre 2021)

115 Camera dei deputati, Servizio Studi, XVIII Legislatura, Scambio di lettere tra l'Italia e la Santa Sede sull'assistenza spirituale alle Forze armate, Dossier n. 356, Scheda di lettura, 23 ottobre 2020, p. 8 (https://documenti.camera.it/Leg18/Dossier/Pdf/ES0249.Pdf - ultima consultazione: 6 novembre 2021).

116 Cfr. L. M. GUZZO, Verso la riforma, cit., pp. 25-26.

117 Sul punto vedi P. CONSORTI, Risposta al Centro Studi dell'Ordinariato militare, in Rivista di Teologia Morale, n. (2001)130, p. 273, che, nel rilevare come la Costituzione 
presentazione del disegno di legge al Senato il relatore, senatore Alessandro Alfieri, ha pure sottolineato che i cappellani non sarebbero più stati "militari tra i militari"118, richiamando la ridefinizione del "concetto di assimilazione ai gradi rispetto al precedente rapporto con le strutture e le funzioni militari"119. In realtà, come abbiamo sopra dimostrato, non cambia la condizione giuridica dei cappellani militari, perché la loro figura è sempre stata "assimilata" a quella dei militari e, anzi, è proprio a partire da questa assimilazione che deriva la problematicità della loro stabile integrazione nelle strutture militari. Così come non è stata modificato il rango dell'Ordinario militare: il grado di tenente generale è esattamente corrispondente al livello di comando di generale di corpo d'armata.

In alcuni casi, insomma, è parso che siano stati attuati tentativi di dissimulazione della reale disciplina normativa. Peraltro, nonostante si trattasse di una questione che ha interessato, in diverse occasioni, l'opinione pubblica, il dibattito pubblico sul provvedimento è stato mortificato dalla "segretezza" che ha caratterizzato il testo dell'Intesa fino all'approdo nell'aula parlamentare ${ }^{120}$, a parte qualche anticipazione su $L^{\prime}$ Osservatore Romano ${ }^{121}$ e sulla rivista Adista ${ }^{122}$.

In fin dei conti, le vicende caratterizzanti la legge n. 70 del 2021 manifestano le profonde incertezze sulle linee di politiche ecclesiastica da parte delle forze politiche di maggioranza ${ }^{123}$ che, di volta in volta,

apostolica Spirituali militum curae non stabilisca una "equiparazione assoluta, né tantomeno formale" con le diocesi, evidenzia che l'Ordinariato militare difetta, tra l'altro, di una "tipica istituzione diocesana" quale l'Istituto diocesano per il sostentamento del clero.

118 Senato della Repubblica, atto parlamentare n. 1763, Relazione, p. 3 (https://www.se nato.it/service/PDF/PDFServer/BGT/01150238.pdf/ - ultima consultazione: 6 novembre 2021).

119 Ibidem.

120 Di intesa "segretissima" si parla in Francesco, pecunia non olet? in Partito per la tutela dei Diritti dei militari e delle forze di polizia, 5 maggio 2021 (in https://www. partitodirittimilitari.it/archives/567 - ultima consultazione: 6 novembre 2021).

121 G. DALLA TORRE, Nuova intesa sull'assistenza religiosa ai militari in Italia, in L'Osservatore Romano, 24 febbraio 2018.

122 Cfr. L. KOCCI, Cappellani militari abili e arruolati. Intesa Italia-Santa Sede sui preti soldato, in Adista. Notizie, n. 7/24 febbraio 2018. ID., Cappellani sì, militari no. Pax Christi e Noi siamo Chiesa contestano l'intesa, in Adista. Notizie, n. 7/24 febbraio 2018; ID., Questa intesa non s'ha da fare. Appello di Pax Christi contro l'accordo sui cappellani militari, in Adista. Notizie, n. 11/24 marzo 2018.

123 Cfr. G. CASUSCELLI, Il "vento del cambiamento" e il "soffio dello spirito", in Stato, Chiese e pluralismo confessionale, cit., n. 23 del 2018, p. 1. 
sostengono i diversi esecutivi nell'imprimere l'indirizzo politico al Paese. Le trattative con la Santa Sede si sono avviate nel marzo 2015, sotto il governo Renzi, quando è stata annunciata l'istituzione di una Commissione paritetica, convocata su richiesta del Governo italiano a gennaio $2016^{124}$. Queste trattative sono poi proseguite durante il governo Gentiloni, il quale ha firmato l'Accordo il 18 febbraio 2018, a pochi giorni dalle elezioni politiche del 4 marzo 2018. L'Intesa è poi rimasta per lungo tempo "nel cassetto"125, anche se nel primo Governo Conte il titolare del dicastero della Difesa, Elisabetta Trenta, parlava dell'Intesa come di un'occasione per "rivisitare l'ordinamento dei cappellani militari"126. La situazione si è sbloccata con il secondo governo Conte, a trazione Movimento 5 Stelle - Partito Democratico, con il Ministro degli affari esteri e della cooperazione internazionale Luigi Di Maio che il 10 marzo 2020 ha presentato al Senato il disegno di legge contenente l'Intesa in questione, per arrivare all'approvazione finale nel marzo 2021, quando da poche settimane iniziava l'avventura del governo Draghi.

Il disegno di legge ha trovato un'accoglienza bipartisan nelle aule parlamentari. Un esempio può essere rappresentato dai lavori nell'aula del Senato il 9 settembre 2020. Per il senatore Alessandro Alfieri, nella sua qualità di relatore, l'Intesa in questione permette di "aggiornare" lo status dei cappellani militari. Subito dopo, la senatrice Antonella Faggi, del partito "Lega - Salvini Premier, ha sottolineato l'esigenza di "ridefinire i profili giuridici di un nuovo rapporto tra i cappellani e la struttura". E lamentandosi, poi, del brusio in aula ha aggiunto: "stiamo per votare una ratifica che probabilmente non interessa a nessuno". Poi, il viceministro degli affari esteri Marina Sereni, in quota Partito Democratico, con un passato nella Federazione Giovanile Comunista, ha sottolineato:

"L'Intesa raggiunge un punto di equilibrio tra l'obiettivo italiano di razionalizzare e contenere gli oneri e la volontà vaticana di preservare alcune prerogative dei cappellani militari. Da qui scaturisce l'interesse del Governo italiano a completare il processo di ratifica"127.

${ }^{124}$ Cfr. A. DE OTO, Il servizio di assistenza spirituale, cit., p. 116.

${ }^{125}$ A. DE OTO, Il servizio di assistenza spirituale, cit., p. 116.

126 Vedi le dichiarazione dell'allora ministro Elisabetta Trenta In arrivo taglio cappellani militari, Adnkronos, 29 novembre 2018 (https://www.adnkronos.com/in-arrivo-taglio-cappellanimilitari-zanotelli-aboliteli_4ILOFhSosNLW5k3gRSgVeN - ultima consultazione: 6 novembre 2021).

127 Per la trascrizione di questi lavori parlamentari vedi il Fascicolo Iter DDL S. 1763, 31 ottobre 2021, pp. 163-165, in https://www.senato.it/leg/18/ BGT/Schede/FascicoloSchedeDDL/ 
La discussione al Senato, che abbiamo brevemente presentato, dà conto di come sull'Intesa le diverse forze politiche, di maggioranza e di minoranza, abbiano trovato un'inedita convergenza, al punto che nell'aula di Villa Madama il provvedimento è stato approvato senza voti contrari, con solo dieci astenuti ${ }^{128}$. In più, il 24 marzo 2021 l'Ufficio Comunicazione del gruppo Movimento 5 Stelle alla Camera dei deputati ha rivendicato i meriti per la ratifica dell'accordo tra Santa Sede e Governo italiano in materia di assistenza spirituale ${ }^{129}$, che sarebbe avvenuta in via definitiva, alla Camera dei deputati il 14 aprile 2021, con solo quattro astenuti ${ }^{130}$ e tre voti contrari ${ }^{131}$.

La legge n. 70 del 2021, con la revisione dell'assistenza religiosa militare per i cattolici ha messo davvero d'accordo tutti. Ci sarebbe da domandarsi se questa convergenza di veduta nel Parlamento italiano rappresenti effettivamente l'ethos e il comune sentire dei cittadini italiani. Non è questa la sede per rispondere a un simile interrogativo. Ma ci limitiamo soltanto a sottolineare che, nel rispetto del divieto del mandato imperativo (art. 67 Cost.), ogni legge dovrebbe in qualche riflettere i valori e le esigenze del gruppo sociale, traducendoli in termini giuridici, in relazione a un determinato periodo storico (ciò - sia chiaro - è cosa ben diversa dall'attuare politiche di stampo populistico). Ne va, altrimenti, dell'efficacia della legge stessa.

Chi si attendeva (legittimamente) che l'approdo (finalmente) all'accordo bilaterale avrebbe rappresentato l'occasione per un ripensamento generale dell'istituto, non può che rimanere deluso. Lo schema logico di una disciplina del tutto costruita su un'integrazione stabile dei cappellani militari, cioè dei sacerdoti cattolici, nella organizzazione militare è, in fin dei conti, rimasto lo stesso, tra vecchio e

ebook/52864.pdf (ultima consultazione: 6 novembre 2021).

128 Alberto Airola (Movimento 5 Stelle), Emma Bonino (Più Europa; gruppo Misto), Andrea Cioffi (Movimento 5 Stelle), Giovanbattista Fazzolari (Fratelli d'Italia), Gianluca Ferrara (Movimento 5 Stelle), Maurizio Gasparri (Forza Italia), Gabriele Lanzi (Movimento 5 Stelle), Carlo Martelli (Italexit; gruppo Misto), Barbara Masini (Forza Italia), Michele Montevecchi (Forza Italia).

${ }^{129}$ Cfr. il comunicato pubblicato sul sito internet Non solo marescialli (al link https://non solomarescialli.it/difesa-m5s-importante-ratifica-con-vaticano-per-riforma-ruolo-cappellani-milita $r /$ - ultima consultazione: 6 novembre 2021).

${ }^{130}$ Nicola Fratoianni (Leu), Sandra Savino (Forza Italia), Vittorio Sgarbi (I Libertari) e Luca Squeri (Forza Italia).

${ }^{131}$ Roberto Giachetti (Italia Viva), Elisa Siragusa (Movimento 5 Stelle) e Riccardo Magi (Più Europa). 
nuovo regime. Anzi, tramite l'Intesa, considerata la sua natura di atto internazionalistico, sono state introdotte nell'ordinamento interno alcune norme in materia che hanno una particolare forza passiva all'abrogazione perché di adeguamento a obbligazioni internazionali. Ne deriva che non soltanto l'istituto dell'assistenza religiosa alle Forze armate rimane invariato nella logica ispiratrice che presiede la materia, ma, ancora di più, questa logica è ormai assunta a un livello sovraordinato rispetto alle norme di rango ordinario. La Chiesa cattolica ha ottenuto il massimo che poteva ottenere da una negoziazione bilaterale con lo Stato italiano, in termini di privilegi ormai anacronistici ${ }^{132}$.

E singolare che il giudizio dottrinale offerto da Tozzi, quasi quarant'anni addietro, in relazione all'art. 1 della legge n. 512 del $1961^{133}$ non perda di attualità:

"[...] l'adozione della nuova legge non ha determinato nemmeno la caducazione della elencazione delle mansioni contenuta nel regio decreto n. 474 del 1936. Parimenti resta ferma ogni precedente indicazione circa la concorrenza fra giurisdizione civile e giurisdizione ecclesiastica, nonché la valenza dell'ufficio dei cappellani, a cavallo fra l'impiego civile e la soggezione ad aspetti della disciplina militare. Nel complesso, insomma, resta immutato l'intero impianto dell'organizzazione del servizio [...]. È singolare che il legislatore del 1961 non abbia avvertito la minima esigenza di adeguamento del vecchio sistema ai mutati principi, che fin dal 1948 avevano notevolmente innovato l'intero quadro di riferimento [...] nel principio enunciato dall'art. 1 della legge n. 512 del 1961 è ancora del tutto assente la prospettiva della soddisfazione dei bisogni del corpo sociale, cioè dei cittadini fedeli, sia in quanto singole persone, sia come appartenenti ad una aggregazione, come la Confessione religiosa, la quale non pretende di esaurire da sola la totalità degli interessi dei suoi componenti"134.

Come si può ben vedere, queste parole possono essere riproposte, senza grandi difficoltà, oggi con riferimento alla legge n. 70 del 2021. Nel frattempo, ci sono state tappe normativamente significative, come

132 Anche G DALlA TORRE, Lezioni di Diritto ecclesiastico, cit., p. 326, metteva in guardia dai "pericoli di nuove fattispecie di giurisdizionalismo che tali forme di integrazione stabile potrebbero comportare".

${ }^{133}$ La disposizione così recitava: "Il servizio dell'assistenza spirituale alle Forze armate dello Stato, istituito per integrare, secondo i principi della religione cattolica, la formazione spirituale delle Forze armate stesse, è disimpegnato da sacerdoti cattolici in qualità di cappellani militari".

${ }^{134}$ V. TOZZI, Assistenza religiosa e diritto ecclesiastico, Jovene, Napoli, 1985, p. 42. 
l'implementazione dell'Accordo del 1984, la codificazione dell'ordinamento militare e, per ultimo, l'Accordo bilaterale tra Governo italiano e Santa Sede, ma l'impianto strutturale della disciplina dell'assistenza religiosa alle Forze armate rimane fermo al periodo prerepubblicano, specie per ciò che riguarda lo statuto giuridico dei cappellani militari cattolici. I relativi dubbi di legittimità costituzionale della disciplina, che già si si avanzavano in riferimento alla legge n. 512 del $1961^{135}$, non stati sciolti e anzi continuano a riproporsi.

La lezione di Giuseppe Tomasi di Lampedusa nel suo Gattopardo rimane sempre attuale: "Se vogliamo che tutto rimanga come è, bisogna che tutto cambi"136.

135 V. TOZZI, Assistenza religiosa, cit., p. 41.

${ }^{136}$ G. TOMASI DI LAMPEDUSA, Il Gattopardo, Feltrinelli, Milano, 2002, p. 50 (prima edizione: 1957). 